\title{
PIECEWISE SELF-SIMILAR SOLUTIONS AND A NUMERICAL SCHEME FOR SCALAR CONSERVATION LAWS*
}

\author{
YONG-JUNG $\mathrm{KIM}^{\dagger}$
}

\begin{abstract}
The solution of the Riemann problem was a building block for general Cauchy problems in conservation laws. A Cauchy problem is approximated by a series of Riemann problems in many numerical schemes. But, since the structure of the Riemann solution holds locally in time only, and, furthermore, a Riemann solution is not piecewise constant in general, there are several fundamental issues in this approach such as the stability and the complexity of computation.

In this article we introduce a new approach which is based on piecewise self-similar solutions. The scheme proposed in this article solves the problem without the time marching process. The approximation error enters in the step for the initial discretization only, which is given as a similarity summation of base functions. The complexity of the scheme is linear. Convergence to the entropy solution and the error estimate are shown. The mechanism of the scheme is introduced in detail together with several interesting properties of the scheme.
\end{abstract}

Key words. self-similarity, characteristics, front tracking, gridless scheme

AMS subject classifications. 65M25, 35L65

PII. S0036142901381364

1. Introduction. Self-similarity of the Cauchy problem for one-dimensional conservation laws,

$$
\begin{aligned}
& v_{t}+f(v)_{x}=0, \quad x, v \in \boldsymbol{R}, t>0 \\
& v(x, 0)=v_{0}(x),
\end{aligned}
$$

with Riemann initial data

$$
v(x, 0)= \begin{cases}v_{-}, & x \leq 0 \\ v_{+}, & x>0\end{cases}
$$

has been the basis of various schemes devised for general initial value problems; see Glimm [10] and Godunov [11], for example. The self-similarity of the Riemann problem is the property that the solution is a function of the self-similarity variable $\xi=x / t$. In other words, the solution is constant along the self-similarity lines

$$
\frac{x}{t}=\text { constant. }
$$

The basic idea of the Godunov scheme for a general initial value problem is to approximate the initial data by a piecewise constant function and then apply the self-similarity structure to the series of Riemann problems.

There are two basic issues we have to consider immediately in this approach. First, since the self-similarity for a piecewise constant solution holds locally in time only, the structure of the Riemann solution can be applied for a small time period. In other words, the scheme is not free from the CFL condition (see [4], [5]), and,

\footnotetext{
*Received by the editors June 7, 2001; accepted for publication (in revised form) July 8, 2002; published electronically December 13, 2002. This work was supported in part by Korea Science and Engineering Foundation (grant R11-2002-103).

http://www.siam.org/journals/sinum/40-6/38136.html

${ }^{\dagger}$ School of Mathematics, University of Minnesota, Minneapolis, MN 55455 (yongkim@math.umn. edu).
} 
hence, the scheme can march just a little amount of time every time step and it costs computation time. Furthermore, since rarefaction waves appear immediately, the solution is not piecewise constant anymore. So a numerical scheme contains a process which rearranges the rarefaction wave into a piecewise constant function every time step. The numerical viscosity enters in this process, and tracking down the behavior of the scheme becomes extremely hard.

LeVeque [20] considers a large time step technique based on the Godunov method for the genuinely nonlinear problem. In the scheme the CFL number may go beyond 1 , and it is even possible to solve the propagation of a simple wave in a single step, i.e., $\Delta t=T$ for the given final time $T>0$. However, the scheme handles interactions between waves incorrectly if the CFL number is so large.

One way to avoid the rearranging process is to consider a modified equation,

$$
\begin{aligned}
& u_{t}+h(u)_{x}=0, \quad x, u \in \boldsymbol{R}, t>0 \\
& u(x, 0)=u_{0}(x),
\end{aligned}
$$

where $h$ and $u_{0}$ approximate $f$ and $v_{0}$, respectively. Dafermos [7] considers a polygonal approximation $h \sim f$, i.e., $h$ is continuous and piecewise linear. In this case the exact solution of (1.4) is piecewise constant. So the method does not require a rearranging process, and, therefore, the numerical viscosity is not introduced and the error is controlled by refining the polygonal approximation $h$. In this approach, the exact behavior of the numerical solution can be monitored more closely and we may get a more detailed understanding of the scheme. This idea has been developed in Holden and Holden [12], and it has been extended to multidimensional problems in Holden and Risebro [14] and to systems of conservation laws in Holden, Lie, and Risebro [13]. In particular, we refer to Bressan [2], [3] for systems. This front tracking method has been developed as a computational tool (e.g., [21], [22]).

Lucier [24] approximates the actual flux $f$ by a piecewise parabolic function $h$ and achieves a second order scheme. In this case, the initial data $v_{0}(x)$ are approximated by a piecewise linear function $u_{0}$ and the solution remains piecewise linear. The difference between the solutions of the original problem (1.1) and the modified problem (1.4) is estimated by

$$
\|v(\cdot, t)-u(\cdot, t)\|_{1} \leq\left\|v_{0}-u_{0}\right\|_{1}+t\left\|f^{\prime}-h^{\prime}\right\|_{\infty}\left\|v_{0}\right\|_{B V} .
$$

Since the linear approximation is of second order, he achieves a second order scheme for a fixed time $t>0$.

If we want to design a numerical scheme which represents the exact solution, we have to find a way to choose grid points correctly. If they are simply fixed, it is clear that the scheme cannot represent the exact solution and, hence, we need to rearrange the solution to fit the solution to the fixed grid points. So it is natural to consider the moving mesh method; see Miller [25]. In Lucier [24] the moving mesh method is used to find the exact solution of (1.4), where mesh points move along characteristics. Another option is not to use any grid point. In numerical schemes based on the front tracking method mentioned earlier, grid points are used just for the initial discretization. The scheme we develop in this article does not use any grid point either.

This article has two goals. The first one is to introduce the mathematical idea which is behind the piecewise self-similar solutions. The second one is to demonstrate how to implement the idea into a numerical scheme and show properties of the scheme. From the study of the Burgers equation (see [17] or Whitham [26]), it is well known 
that the primary structure which dominates the evolution is a saw-tooth profile. In fact, this profile is a series of $\mathrm{N}$-waves and eventually the solution evolves to a single $\mathrm{N}$-wave; see Liu and Pierre [23]. The starting point of our scheme is to use this structure as the unit of the scheme.

If a solution $u(x, t)$ is a function of the self-similarity variable $\xi=x / t$, then we can easily derive from (1.1) that

$$
f^{\prime}(u(x, t))=x / t
$$

Roughly speaking, a piecewise self-similarity (initial) profile has the structure of

$$
f^{\prime}(u(x, 0))=\frac{x-c_{k}}{t_{k}}, \quad x \in\left(a_{k}, b_{k}\right), c_{k}, t_{k} \in \boldsymbol{R} .
$$

Note that the time index $t_{k}$ can be a negative number. In this article, we observe that the solution of (1.1) with piecewise self-similarity initial profile has such a structure for all $t>0$, i.e.,

$$
f^{\prime}(u(x, t))=\frac{x-c_{k}}{t+t_{k}}, \quad x \in\left(a_{k}(t), b_{k}(t)\right), c_{k}, t_{k} \in \boldsymbol{R}, t \in \boldsymbol{R}^{+},
$$

and we give the explicit formula for this kind of solution in several situations. First we consider a convex flux with positive wave speed,

$$
f^{\prime \prime}(u) \geq 0, \quad f^{\prime}(u) \geq 0,
$$

where $f$ is locally Lipschitz continuous. The convexity of the flux $f^{\prime \prime}(u) \geq 0$ is used to get the explicit formula $g(x)$ of the self-similarity profile such that $f^{\prime}(g(x))=x$, and the self-similarity profile (1.7) can be written as

$$
u(x, t)=g\left(\frac{x-c_{k}}{t+t_{k}}\right), \quad x \in\left(a_{k}(t), b_{k}(t)\right), c_{k}, t_{k} \in \boldsymbol{R}, t \in \boldsymbol{R}^{+} .
$$

Note that the equality is included for the second derivative of the flux in $(\mathrm{H})$, and, hence, the monotonicity of $f^{\prime}$ is not strict and $g(x)$ is not exactly the inverse function of $f^{\prime}$, and $g\left(f^{\prime}(u)\right) \neq u$ in general. In this approach, we may include a piecewise linear flux of the front tracking method; see Remark 6.4.

Our approach is as follows. We start our discussion reviewing the self-similarity property in conservation laws in section 2. This discussion leads us to the study of piecewise self-similar solutions, which is the case when the self-similarity lines and characteristics are compatible. In section 3 we consider a piecewise self-similar solution which can be written as a self-similarity summation (or simply S-summation),

$$
\bigodot_{k=1}^{n} B_{m_{k}, t_{k}, c_{k}}(x), \quad c_{n}<\cdots<c_{2}<c_{1}
$$

of a finite number of base functions. We give definitions for the S-summation and base functions in the section and show that $u(x, t)=\bigodot_{k=1}^{n} B_{m_{k}, t+t_{k}, c_{k}}(x)$ is the solution of (1.1) with initial data $u_{0}(x)=\bigodot_{k=1}^{n} B_{m_{k}, t_{k}, c_{k}}(x)$; see Theorem 3.6. We consider $u$ as an approximation of the solution $v$ with the original initial data $v_{0}$. Then the $L^{1}$ contraction theory of conservation laws (see Hörmander [15], Kruzhkov [18], [19]) implies

$$
\|v(\cdot, t)-u(\cdot, t)\|_{1} \leq\left\|v_{0}-u_{0}\right\|_{1} .
$$


It is the estimate corresponding to the error estimate (1.5), which does not have the time dependent term anymore. It is natural to expect that the error increases in time if the flux is changed. In our approach, we use the original flux and the error decreases in time. In fact, the left-hand side of (1.10) is of order $O\left(t^{-1}\right)$; see [16]. The convergence of the scheme is now clear (see Theorem 3.6, Corollary 3.7). Note that the S-summation (1.9) represents only a special kind of piecewise self-similar profile in (1.6), which has positive indexes $t_{k}>0$ and is ordered appropriately, i.e., $c_{n}<\cdots<c_{2}<c_{1}$ if $a_{n} \leq b_{n} \leq \cdots \leq a_{2} \leq b_{2} \leq \cdots \leq a_{1} \leq b_{1}$.

The S-summation is successfully coded for a numerical scheme in section 4 . This scheme has several unique properties. First, it does not require a time marching procedure. So the complexity of the scheme is of order $O(N)$, not $O\left(N^{2}\right)$. CPU times for several cases are compared in section 4.3. Second, it captures the shock location very well even if a small number of base functions (or mesh points) are used; see Figure 4.5. In the figure it is clearly observed that the solution with finer mesh always passes through bigger artificial shocks. Since it does not introduce numerical viscosity at all, we obtain a very good resolution for an inviscid problem. This scheme also distinguishes physical shocks and artificial ones clearly. Table 4.4 shows the time when the physical shock appears.

In section 5 we generalize the method. For a general convex flux, i.e.,

$$
f^{\prime \prime}(u) \geq 0,
$$

the method is applied through the transformations (5.1) and (5.3). If the flux has inflection points, then the scheme becomes considerably complicated and it is beyond the purpose of this article. But, if the flux has only one inflection point, for example,

$$
f^{\prime \prime}(u) \leq 0 \text { for } u \leq A, \quad f^{\prime \prime}(u) \geq 0 \text { for } u \geq A,
$$

then we can easily apply the scheme through a similar transformation (5.4). Dafermos [8] considers a flux with a single inflection point through generalized characteristics. The Buckley-Leverett equation satisfies this condition. The flux $f(u)=u^{2}-u^{3}$, which appears in thin film flows (see Bertozzi, Münch, and Shearer [1]), also belongs to this category. Figure 5.2 shows the strength of our scheme over the Godunov scheme in this case.

The scheme is not good enough for a short time behavior $t \ll 1$ since the initial error $\left\|v_{0}-u_{0}\right\|_{1}$ is not controlled efficiently. To resolve this issue we add an extra structure to base functions in section 6 . Using these base functions, we can approximate the initial data with second order accuracy and solve the solution for the modified initial datum. Furthermore, a general piecewise self-similarity profile (1.6) can be written in terms of S-summation of these modified base functions.

2. Self-similarity in conservation laws. Consider one-dimensional scalar conservation laws,

$$
\begin{aligned}
& u_{t}+f(u)_{x}=0, \\
& u(x, 0)=u_{0}(x), \quad x, u \in \boldsymbol{R}, t>0
\end{aligned}
$$

where the flux $f$ is locally Lipschitz continuous. For a nonlinear flux $f(u)$ the solution may have a singularity, and hence the solution is considered in the weak sense with the entropy admissibility condition:

$$
\frac{f(\tilde{u})-f\left(u_{-}\right)}{\tilde{u}-u_{-}} \geq \frac{f\left(u_{+}\right)-f\left(u_{-}\right)}{u_{+}-u_{-}}
$$


for any number $\tilde{u}$ lying between $u_{+}=u(x+, t)$ and $u_{-}=u(x-, t)$. It is well known that the self-similarity of a conservation law is inherited from the fact that a rescaled function,

$$
w(x, t)=u(a x, a t), \quad a>0,
$$

is also the solution of (2.1) if and only if the initial profile $u_{0}(x)$ satisfies

$$
u_{0}(x)=u_{0}(a x), \quad a>0 .
$$

It is clear that, if the Riemann initial value,

$$
u(x, 0)= \begin{cases}u_{-}, & x<0, \\ u_{+}, & x>0,\end{cases}
$$

is given, (2.4) is satisfied and, hence, $u(x, t)=u(a x, a t)$ for all $a>0$. Therefore, $u(x, t)$ is a function of the self-similarity variable,

$$
u(x, t)=u(\xi), \quad \xi=x / t .
$$

The structure of a Riemann solution is given in Figure 2.1 together with characteristic lines. Note that, even though a self-similarity line $x / t=\xi, \xi \in \boldsymbol{R}$, is not a characteristic line, the solution is constant along it. This is a special property of the Riemann problem, and it is not expected in a general Cauchy problem.

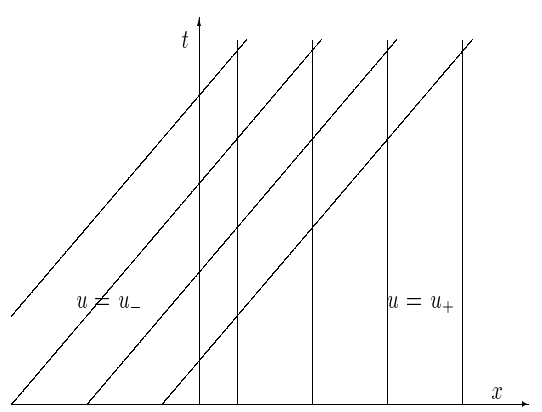

(a) Characteristic lines

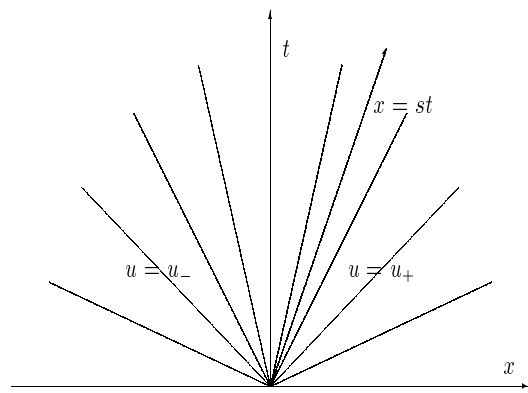

(b) Self-similarity lines

FIG. 2.1. Let $f^{\prime}\left(u_{+}\right)=0$ and $f^{\prime}\left(u_{-}\right)=1$. Then self-similarity lines are different from characteristic lines. However, the solution is constant along self-similarity lines.

If the total mass of the initial data $u_{0}(x)$ is finite, i.e.,

$$
\int\left|u_{0}(x)\right| d x<\infty,
$$

then the relation (2.4) cannot be satisfied since the transformation $u_{0}(x) \rightarrow u_{0}(a x)$ does not preserve the total mass. So the solution cannot be a function of self-similarity variable $\xi=x / t$. In the following, we consider techniques to achieve the Riemann solution like self-similarity for general Cauchy problems.

Suppose that characteristic lines of the solution $u(x, t)$ pass through the origin. Then the relation between the wave speed and characteristics gives

$$
f^{\prime}(u)=\frac{x}{t} .
$$


Since the right-hand side diverges as $t \rightarrow 0$, we consider the initial datum as the profile at a given time $t_{0}>0$. The simplest case of $L^{1}$ initial datum of the kind is

$$
f^{\prime}(u(x, 0))=\frac{x}{t_{0}} \text { if } 0<x<s_{0}, \quad u(x, 0)=0 \text { otherwise. }
$$

Characteristic lines of this initial profile are given in Figure 2.2. Nonvertical characteristics pass through the point $\left(0,-t_{0}\right)$, and there is a region in which characteristic lines overlap with each other. The solution is given by finding the shock characteristic $x=s(t)$ correctly. In this case, the shock characteristic $x=s(t)$ is not a straight line and the solution is not a function of $x /\left(t+t_{0}\right)$. However, the solution is a function of $x /\left(t+t_{0}\right)$ in the region $0<x<s(t)$, i.e.,

$$
f^{\prime}(u(x, t))=\frac{x}{t+t_{0}} \text { if } 0<x<s(t), \quad u(x, t)=0 \quad \text { otherwise. }
$$

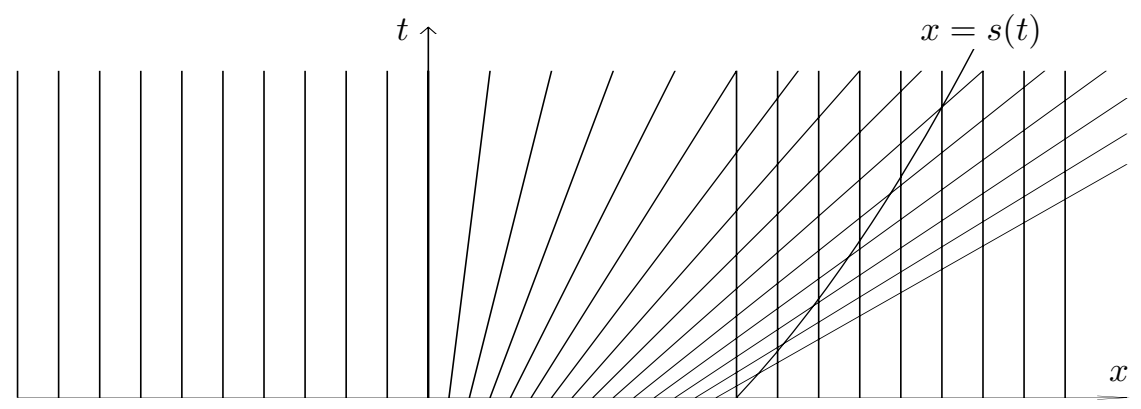

FIG. 2.2. Characteristic lines of a self-similarity solution are similar to self-similarity lines. The main difference is that the shock characteristic is not a straight line anymore.

Since the shock speed $s^{\prime}(t)$ satisfies the Rankine-Hugoniot jump condition, the shock location $s(t)$ can be found by its integral form. On the other hand, if the convexity of the flux $f$ is assumed, i.e.,

$$
f^{\prime \prime}(u) \geq 0
$$

we may consider the self-similarity profile $g(x)$ such that $f^{\prime}(g(x))=x$. In this case we obtain

$$
u(x, t)=g\left(x /\left(t+t_{0}\right)\right), \quad 0<x<s(t),
$$

and we can easily find the shock location $s(t)$ using the equal area rule,

$$
\int_{0}^{s(t)} g\left(x /\left(t+t_{0}\right)\right) d x=\int_{0}^{s_{0}} g\left(x / t_{0}\right) d x, \quad t>0 .
$$

Since the conservation law (2.1) does not explicitly depend on the $x$ variable, we may translate the initial data $(2.9)$ in the $x$-direction. We can also consider initial data which consist of a finite number of structures in (2.9). A simple example is

$$
u_{0}(x)=\sum_{k=1}^{N} g\left(\frac{x-c_{k}}{t_{k}}\right) \chi_{\left(c_{k}, s_{k}\right)},
$$


where centers $c_{k}$ and shock locations $s_{k}$ satisfy

$$
-\infty<c_{N}<s_{N}<\cdots<c_{1}<s_{1}<\infty .
$$

The time indexes $t_{k}>0$ in (2.13) decide the slope of the initial profile, and they do not need to be identical. Condition (2.14) implies that all the profiles in (2.13) are separated. If not, the simple summation in (2.13) breaks down the selfsimilarity structure we want to keep. In section 3 we consider an S-summation which preserves this structure. Figure 2.3 shows characteristic lines for initial data (2.13) with $N=4$. In this case, tracking down a shock is more complicated and (2.12) is not valid anymore. The following section is devoted to handling the general case.

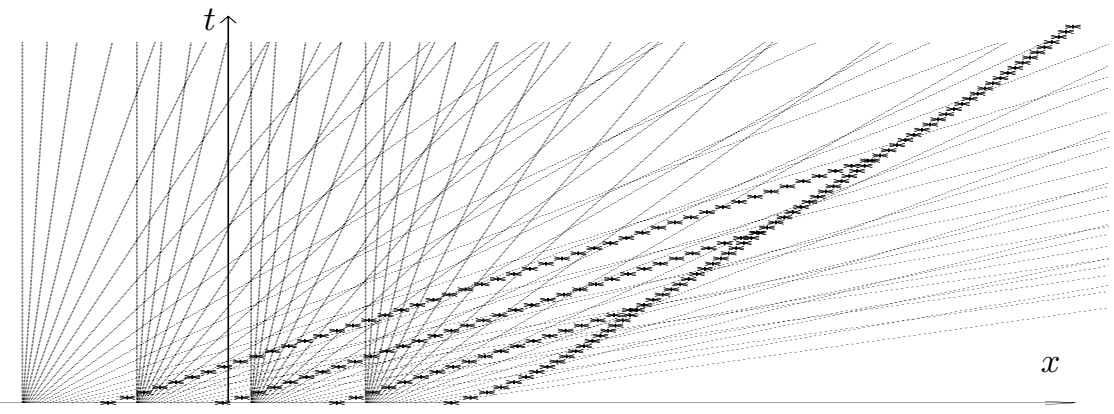

FIG. 2.3. Shock characteristics (dots) are merged together after contacts among them, and a bigger shock appears which is a physical one.

3. Piecewise self-similar solutions. In this section we define the S-summation and show that, if the initial value $u_{0}(x)$ is given as an S-summation, then so is the solution $u(x, t)$ of $(2.1)$ at any given time $t>0$. We consider the flux under the hypothesis,

$$
f^{\prime \prime}(u) \geq 0, \quad f^{\prime}(u) \geq 0,
$$

and the self-similarity profile $g(x)$ satisfies $f^{\prime}(g(x))=x$. We may assume $f^{\prime}(0)=0$ without the loss of generality, and it implies that the solution is actually assumed to be positive under $(\mathrm{H})$. The results in this section are generalized in section 5 .

3.1. Base functions. As was mentioned earlier, the self-similarity profile

$$
u(x, t)=g(x / t), \quad t>0,
$$

represents the asymptotic behavior of the conservation law (1.1). A triple index function $B_{t, c, s}(x)$, defined by

$$
B_{t, c, s}(x)=\left\{\begin{array}{cl}
g((x-c) / t), & c<x<s, \\
0, & \text { otherwise }
\end{array}\right.
$$

serves as a base function in this article. A base function has the self-similarity profile over the interval between the center $c$ and the shock location s. The area (or mass) $m$ enclosed by the $x$-axis and the base function is given by

$$
m=\int_{c}^{s} B_{t, s, c}(x) d x=\int_{0}^{s-c} g(x / t) d x=: m(t, c, s) .
$$


It is convenient to consider the mass $m$ as the fourth index of the base function, say, $B_{m, t, c, s}(x)$, or any three of them as an index set. In any case we always assume that indexes $m, t, c, s$ satisfy the relation (3.3), i.e., if any three of them are given, the fourth one is decided by the relation.

Consider a Cauchy problem,

$$
\begin{gathered}
u_{t}+f(u)_{x}=0, \\
u(x, 0)=B_{m_{0}, t_{0}, c_{0}, s_{0}}(x) .
\end{gathered}
$$

It is already observed in $(2.10)$ that the solution $u(\cdot, t)$ has the self-similarity profile with time index $t+t_{0}$ between the original center $c_{0}$ and a new shock location $s(t)$. Since the initial total mass $m_{0}$ should be preserved, the solution of (3.4) is

$$
u(x, t)=B_{m_{0}, t+t_{0}, c_{0}}(x),
$$

where the shock location $x=s(t)$ is decided by the relation (3.3).

Remark 3.1. If we take a $\delta$-function as the initial datum, for example, $u_{0}(x)=$ $m_{0} \delta\left(x-c_{0}\right)$, then the solution is $u(x, t)=B_{m_{0}, t, c_{0}}(x)$. So the slope of the base function represents the time of the evolution starting from the $\delta$-function-like initial data, and that is why we take index $t$ for the base function.

Remark 3.2. For the Burgers case, $f(u)=u^{2} / 2$, the self-similarity profile is the identity function, $g(x)=x$. In this case, (3.3) gives explicit relations,

$$
m=(s-c)^{2} /(2 t), \quad t=(s-c)^{2} / 2 m, \quad s=c+\sqrt{2 m t}, \quad c=s-\sqrt{2 m t} .
$$

Remark 3.3. The rescaling (2.3) does not preserve the total mass. So it can not measure the invariance property for $L^{1}$ solutions of conservation laws. For the Burgers case, $f(u)=u^{2} / 2$, we may consider

$$
v(x, t)=a u\left(a x, a^{2} t\right), \quad a>0,
$$

where the rescaling preserves the total mass. We can easily check that variables

$$
w=\sqrt{t+t_{0}} u, \quad \zeta=\left(x-c_{0}\right) / \sqrt{t+t_{0}}, \quad \tau=\ln \left(t+t_{0}\right),
$$

are invariant under the rescaling after the translation $x-c_{0} \rightarrow x, t+t_{0} \rightarrow t$. These variables are called self-similarity variables for $L^{1}$ Cauchy problems, and the Cauchy problem (3.4) is transformed to

$$
\begin{gathered}
w_{\tau}+\frac{1}{2}\left(w^{2}-\zeta w\right)_{\zeta}=0, \\
w\left(\zeta, \ln \left(t_{0}\right)\right)=B_{m_{0}, t_{0}=1, c_{0}=0}(\zeta) .
\end{gathered}
$$

We can easily check that $B_{m_{0}, t_{0}=1, c_{0}=0}(\zeta)$ is an admissible steady state of the equation, and hence $w(\zeta, \tau)=B_{m_{0}, t_{0}=1, c_{0}=0}(\zeta)$ is the solution of (3.9). If we transform the variables back to $u, t, x$, then we get $u(x, t)=B_{m_{0}, t+t_{0}, c_{0}=0}(x)$. This is another way to show (3.5). In this example we can see that the approach with piecewise self-similar solutions captures the self-similarity of the general Cauchy problems exactly. For a detailed study of the transformed problem (3.9), we refer to [17].

3.2. S-summation. Since the solution of (3.4) is given by (3.5), we can easily guess that

$$
u(x, t)=\sum_{k=1}^{n} B_{m_{k}, t_{k}+t, c_{k}}(x)
$$


is the solution of the conservation law with initial data

$$
u_{0}(x)=\sum_{k=1}^{n} B_{m_{k}, t_{k}, c_{k}}(x), \quad c_{n}<\cdots<c_{2}<c_{1},
$$

if all the supports of the base functions in (3.10) are disjoint. But it is not usually the case since the support of a base function expands in time. The S-summation,

$$
B_{n}(x)=\bigodot_{k=1}^{n} B_{m_{k}, t_{k}, c_{k}}(x), \quad c_{n}<\cdots<c_{2}<c_{1},
$$

is to handle the case that supports of base functions overlap with each other. The definition is given inductively in the following.

Let $B_{1}(x)=B_{m_{1}, t_{1}, c_{1}}(x)$. Suppose that $B_{j-1}(x)=\bigodot_{k=1}^{j-1} B_{m_{k}, t_{k}, c_{k}}(x), j \leq$ $n$, is well defined, $\operatorname{supp}\left(B_{j-1}\right) \subset\left[c_{j-1}, \infty\right)$, and that $\int_{c_{j-1}}^{\infty} B_{j-1}(x) d x=\sum_{k=1}^{j-1} m_{k}$. Consider a point $\xi_{j} \in \boldsymbol{R}$ such that $c_{j}<\xi_{j}$,

$$
\begin{gathered}
g\left(\left(x-c_{j}\right) / t_{j}\right)>B_{j-1}(x), \quad c_{j}<x<\xi_{j}, \\
\int_{c_{j}}^{\xi_{j}} g\left(\left(x-c_{j}\right) / t_{j}\right) d x+\int_{\xi_{j}}^{\infty} B_{j-1}(x) d x=\sum_{k=1}^{j} m_{k} .
\end{gathered}
$$

Under assumption (3.13), the left-hand side of (3.14) is monotone in $\xi_{j}$ and, hence, such a point is unique. If there exists such a point $\xi_{j}>c_{j}$, we define

$$
B_{j}(x)=\bigodot_{k=1}^{j} B_{m_{k}, t_{k}, c_{k}}(x)=\left\{\begin{array}{cc}
g\left(\left(x-c_{j}\right) / t_{j}\right), & c_{j}<x<\xi_{j}, \\
B_{j-1}(x), & \text { otherwise }
\end{array}\right.
$$

Clearly, $\operatorname{supp}\left(B_{j}\right) \subset\left[c_{j}, \infty\right)$ and $\int_{c_{j}}^{\infty} B_{j}(x) d x=\sum_{k=1}^{j} m_{k}$, and we may continue the inductive argument. If not, the S-summation (3.12) is not defined.

Base functions are ordered by centers $c_{k}$, and then the S-summation is given from the right-hand side. It is because of the positiveness assumption for the wave speed, $f^{\prime}(u) \geq 0$, in $(\mathrm{H})$. If the order of the summation is changed, the result is different. So the S-summation is not associative.

Remark 3.4. If the time indexes are identical, $t_{k}=t_{0}$, for all $k$, then we can show the S-summation (3.12) is well defined. Then, since the self-similarity profile $g(x)$ is an increasing function, we have $g\left(\left(x-c_{j}\right) / t_{0}\right)>g\left(\left(x-c_{k}\right) / t_{0}\right)$ for all $k<j$. Since $B_{j-1}(x)$ has values of $g\left(\left(x-c_{k}\right) / t_{0}\right), k<j$, piecewise, the inequality (3.13) is satisfied for all $\xi_{j}>c_{j}$. Furthermore the left-hand side of (3.14) has value $\sum_{k=1}^{j-1} m_{k}$ for $\xi_{j}=c_{j}$ and diverges to $\infty$ as $\xi_{j} \rightarrow \infty$. So there exists a point $\xi_{j}$ satisfying (3.14), and the S-summation is well defined.

Remark 3.5. We may consider $\xi_{j}$ as the location of the $j$ th (artificial) shock generated by the base function $B_{m_{j}, t_{j}, c_{j}}$. Suppose that $\xi_{j-1}<\xi_{j}$, i.e., the $j$ th shock caught up the $(j-1)$ st shock. The definition (3.15) implies that the self-similarity profile $g\left(\left(x-c_{j-1}\right) / t_{j-1}\right)$ disappears. We can easily check that we will get the same S-summation (3.15) if we remove the $(j-1)$ st base function and increase $m_{j}$ by adding $m_{j-1}$. This property represents the irreversibility of conservation laws and plays the key role in the numerical scheme (see section 4.2, Step 2). 
THEOREM 3.6. Suppose that $f^{\prime \prime}(u) \geq 0$ and $f^{\prime}(u) \geq 0$. If the S-summation $u_{0}(x) \equiv \bigodot_{k=1}^{n} B_{m_{k}, t_{k}, c_{k}}(x)$ is well defined, then $u(x, t) \equiv \bigodot_{k=1}^{n} B_{m_{k}, t_{k}+t, c_{k}}(x)$ is also well defined for all $t>0$ and it solves (1.1) with its initial value $u_{0}(x)$. If $v(x, t)$ is the entropy solution of (1.1) with its initial value $v_{0} \in L^{1}$, then

$$
\|v(\cdot, t)-u(\cdot, t)\|_{1} \leq\left\|v_{0}-u_{0}\right\|_{1} .
$$

Proof. We may assume $f^{\prime}(0)=0$ without the loss of generality. The proof is completed through inductive arguments. In section 2 , we have shown the theorem for $n=1$. Now we show the theorem for $n=j>1$ assuming that it holds for $n=j-1$. Note that, from the definition, the S-summation $\bigodot_{k=1}^{i} B_{m_{k}, t_{k}, c_{k}}(x)$ is well defined for any $i \leq n$.

Let $u_{j-1}(x, t)$ be the solution of (1.1) with its initial value $\bigodot_{k=1}^{j-1} B_{m_{k}, t_{k}, c_{k}}(x)$. From the assumption, $u_{j-1}(x, t)=\bigodot_{k=1}^{j-1} B_{m_{k}, t_{k}+t, c_{k}}(x)$. Let $u_{j}(x, t)$ be the solution with $u_{j}(x, 0)=\bigodot_{k=1}^{j} B_{m_{k}, t_{k}, c_{k}}(x)$ and $x=\xi_{j}(t)$ be the shock characteristic given by the $j$ th base function, i.e., $\xi_{j}(0)$ is the same as the $\xi_{j}$ in (3.13)-(3.14). Consider a backward characteristic, associated with $u_{j}(x, t)$, that emanates from a point $(x, t)$, $x>\xi_{j}(t)$. From the admissibility of the shock, it does not interact with $x=\xi_{j}(\tau)$, $\tau<t$, and, hence, it is actually the one associated with $u_{j-1}(x, t)$. So we have $u_{j}(x, t)=u_{j-1}(x, t)$.

For $x<c_{j}, u_{j}(x, t)=0$ since the (vertical) forward characteristic that emanates from a point $(x, 0), x<c_{j}$, does not intersect with shock characteristics which move to the right-hand side under the assumption $f^{\prime}(u) \geq 0$. The backward characteristic that emanates from a point $(x, t), c_{j}<x<\xi_{j}(t)$, is a straight line connecting $\left(c_{j},-t_{j}\right)$ since the initial profile over the interval $\left(c_{j}, \xi_{j}(0)\right)$ is self-similar. Hence, $u_{j}(x, t)=$ $g\left(\left(x-c_{j}\right) /\left(t+t_{j}\right)\right)$ for $c_{j}<x<\xi_{j}(t)$, and the shock location $x=\xi_{j}(t)$ should satisfy

$$
\int_{c_{j}}^{\xi_{j}(t)} g\left(\left(x-c_{j}\right) /\left(t+t_{j}\right)\right) d x+\int_{\xi_{j}(t)}^{\infty} u_{j-1}(x, t) d x=\sum_{k=1}^{j} m_{k}
$$

since the total mass is preserved. So $u_{j}(x, t)=\bigodot_{k=1}^{j} B_{m_{k}, t_{k}+t, c_{k}}(x)$ from the definition of the S-summation, and the first part of the proof is complete. The second part (3.16) is simply the $L^{1}$ contraction theory for conservation laws.

In the proof we employ the theory of characteristics (see [9, Chap. 11]). The error estimate (3.16) implies that the initial error decreases in time. In fact, the error is of order $O\left(t^{-1}\right)$ as $t \rightarrow \infty$ (see [16] for detail). The scheme has ideal properties for the study of asymptotic behavior.

Now we consider $u_{0}(x)=\bigodot_{k=1}^{n} B_{m_{k}, t_{k}, c_{k}}(x)$ as an approximation of $L^{1}$ initial value $v_{0}$. Let a partition $\mathcal{C}=\left\{c_{n}<\cdots<c_{1}\right\}$ be the set of centers. Its norm is defined by $\|\mathcal{C}\|=\max \left|c_{k}-c_{k-1}\right|$. There can be many ways to discretize the initial value. To guarantee the convergence of the scheme, we need the existence of $\delta, L>0$ such that

$$
\left\|v_{0}(x)-u_{0}(x)\right\|_{1} \leq \varepsilon \quad \text { if } \quad\|\mathcal{C}\| \leq \delta \text { and } c_{n}<-L, L<c_{1},
$$

where a constant $\varepsilon>0$ is given. An example of such a discretization is given in section 4.2. The convergence of the scheme satisfying (3.17) is clear from (3.16).

COROLlary 3.7 (convergence). The scheme of the S-summation $u(x, t)=$ $\bigodot_{k=1}^{n} B_{m_{k}, t+t_{k}, c_{k}}(x)$ with initial discretization $u_{0}(x)=\bigodot_{k=1}^{n} B_{m_{k}, t_{k}, c_{k}}(x)$ satisfying (3.17) converges to the entropy solution $v(x, t)$ with initial data $v_{0} \in L^{1}(\boldsymbol{R})$ as $\delta \rightarrow 0, L \rightarrow \infty$. 
Remark 3.8. Now we consider the S-summation between two base functions, $\bigodot_{k=1}^{2} B_{m_{k}, t_{k}, c_{k}}(x), c_{2} \leq c_{1}$ (see Figure 3.1). It gives a good example for figuring out the meaning of the S-summation. Furthermore, in the numerical computation, we can possibly compare only two base functions each time and, hence, it is worth considering it in detail. If these two base functions are separated, i.e., $s_{2}<c_{1}$, then the shock place $\xi$ of the definition (3.15) is simply $\xi=s_{2}$. If $c_{1}<s_{2}$, then $\xi$ satisfies

$$
\int_{c_{2}}^{\xi} g\left(\frac{x-c_{2}}{t_{2}}\right) d x+\int_{\xi}^{\max \left(\xi, s_{1}\right)} g\left(\frac{x-c_{1}}{t_{1}}\right) d x=m_{1}+m_{2}
$$

If $\xi>s_{1}$, (3.15) implies that two base functions are merged, i.e.,

$$
\bigodot_{k=1}^{2} B_{m_{k}, t_{k}, c_{k}}(x)=B_{m_{1}+m_{2}, t_{2}, c_{2}}(x) \quad \text { if } \quad s_{1}<\xi .
$$

For the Burgers case, $f(u)=u^{2} / 2,(3.18)$ implies that the trapezoid $B C s_{2} \xi$ in Figure 3.1 has the same area as the triangle $A c_{1} \xi$.

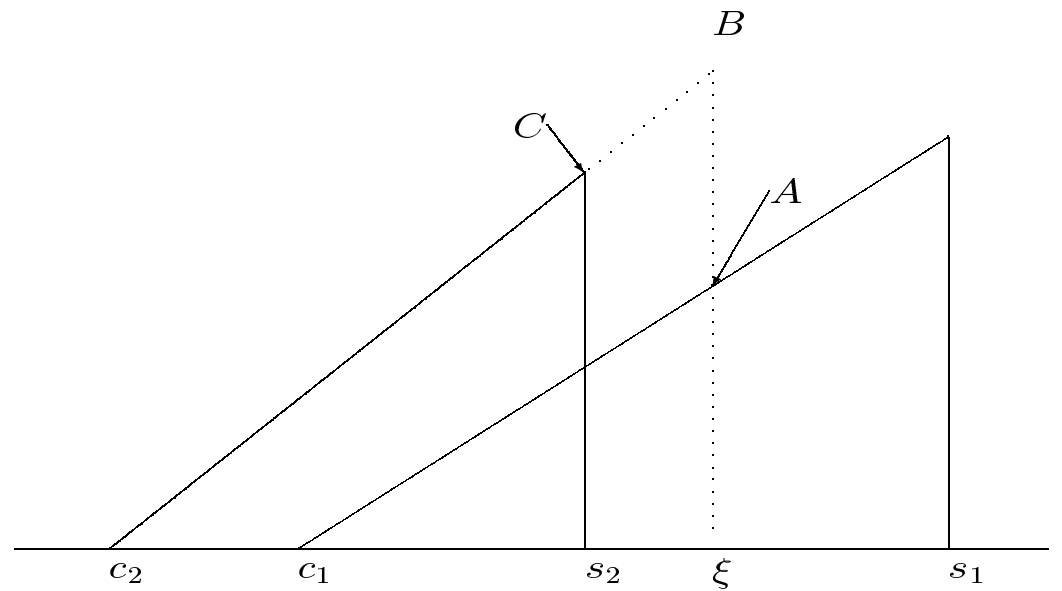

FIG. 3.1. The equal area rule gives the shock location when two base functions interact together.

4. S-summation as a numerical scheme. In this section we show how the S-summation can be implemented into a numerical scheme. We assume that the flux is convex $f^{\prime \prime}(u) \geq 0$ and the solution is positive and compactly supported. More general cases are considered in the following sections. To see what is really happening in each step, we consider a Cauchy problem for the Burgers equation,

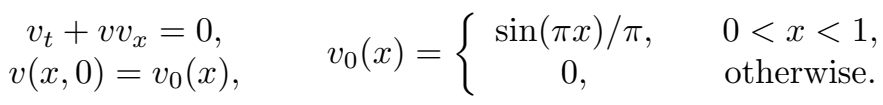

This simple example helps us to visualize the mechanism of the scheme. In section 4.3 we consider more complicated examples and compare CPU times of each computation to check the complexity of the method which is of order $O(N)$. Several properties of this scheme are compared with those of the Godunov method. 
4.1. Preliminaries. First, we consider basic properties of the self-similarity profile $g(x), x>0$. If the flux is strictly convex, $f^{\prime \prime}(u)>0$, then $f^{\prime}(u)$ is invertible and the self-similarity profile is simply the inverse function of $f^{\prime}(u)$. For example, if the flux is given by a power law

$$
f(u)=\frac{1}{\gamma} u^{\gamma}, \quad u \geq 0, \gamma>1,
$$

then the self-similarity profile $g(x)$ is simply

$$
g(x)=\sqrt[\gamma-1]{x}, \quad x>0 .
$$

This is a case that the self-similarity profile is given explicitly. In general, the value of the self-similarity profile $\bar{u}=g(\bar{x})$ at a given point $\bar{x} \geq 0$ is obtained from the basic relation $f^{\prime}(g(\bar{x}))=\bar{x}$, i.e., we need to solve

$$
f^{\prime}(\bar{u})-\bar{x}=0, \quad \bar{u} \geq 0 .
$$

The relation between the self-similarity profile and the wave speed, $f^{\prime}(g(x))=x$, also makes it easy to handle the integrals of a base function. Let $\bar{u}=g(\bar{x} / t)$. Using the change of variables $u=g(x / t)$, we obtain

$$
\int_{0}^{\bar{u}} t f^{\prime}(u) d u=\int_{0}^{\bar{x}} \frac{x}{t} g^{\prime}\left(\frac{x}{t}\right) d x=\bar{x} g\left(\frac{\bar{x}}{t}\right)-\int_{0}^{\bar{x}} g\left(\frac{x}{t}\right) d x .
$$

So the integral of the self-similarity profile is written as a function of $\bar{u}$ or $\bar{x}$ only, i.e.,

$$
\int_{0}^{\bar{x}} g\left(\frac{x}{t}\right) d x=\bar{x} g\left(\frac{\bar{x}}{t}\right)-t f\left(g\left(\frac{\bar{x}}{t}\right)\right)=t \bar{u} f^{\prime}(\bar{u})-t f(\bar{u}) .
$$

Now we consider a simple lemma which is used in deciding the initial time index $t_{0}>0$. This lemma implies that the graph $y=g\left((x-c) / t_{0}\right), x>c$, crosses over $y=v_{0}(x)$ just once.

Lemma 4.1. Suppose that the (smooth and bounded) initial value $v_{0}(x)$ satisfies

$$
v_{0}^{\prime}(x)<\frac{1}{t_{0} f^{\prime \prime}\left(v_{0}(x)\right)} .
$$

Then the point $\bar{x} \geq c$ satisfying $g\left((\bar{x}-c) / t_{0}\right)=v_{0}(\bar{x})$ is unique.

Proof. Differentiating both sides of $f^{\prime}(g(x))=x$, we obtain

$$
g^{\prime}(x)=\frac{1}{f^{\prime \prime}(g(x))}=\frac{1}{f^{\prime \prime}(v)}
$$

where $v=g(x)$. Let $g\left(\left(x_{1}-c\right) / t_{0}\right)=v_{0}\left(x_{1}\right)$ for a point $x_{1} \geq c$. Since

$$
v_{0}^{\prime}\left(x_{1}\right)<\frac{1}{t_{0} f^{\prime \prime}\left(v_{0}\left(x_{1}\right)\right)}=\frac{1}{t_{0}} g^{\prime}\left(\frac{x_{1}-c}{t_{0}}\right)=\partial_{x} g\left(\frac{x_{1}-c}{t_{0}}\right),
$$

we may choose $\delta>0$ such that $g\left((x-c) / t_{0}\right)>v_{0}(x)$ for $x \in\left(x_{1}, x_{1}+\delta\right)$. Now we show that $g\left((x-c) / t_{0}\right)>v_{0}(x)$ for all $x>x_{1}$, which completes the proof. Suppose that $g\left(\left(x_{2}-c\right) / t_{0}\right)=v_{0}\left(x_{2}\right)$ for $x_{2}>x_{1}$. We may take $x_{2}$ as the smallest one. Then $g\left((x-c) / t_{0}\right)>v_{0}(x)$ on $\left(x_{1}, x_{2}\right)$ and it implies $v_{0}^{\prime}\left(x_{2}\right) \geq \partial_{x} g\left(\left(x_{2}-c\right) / t_{0}\right)$. It contradicts the fact that (4.8) holds for $x=x_{2}$. 
4.2. Implementation. Here we introduce a gridless scheme based on the Ssummation.

Step 1 (initial discretization). The first step is to design a method to approximate the initial value $v_{0}(x)$ by an S-summation $u_{0}(x)$ which satisfies (3.17). Consider $n$ base functions $B[k], k=1,2, \ldots, n$. Each element $B[k]$ consists of two members $B[k] . m, B[k] . c$, which represent the mass (or area) and the center of the base function. We use the identical time index $t_{k}=t_{0}$ for all $k$, and, hence, we do not need an extra member for the time index. The first thing to do is to choose the time index $t_{0}>0$ satisfying (4.6). If (4.6) does not hold for any $t_{0}>0$, we need to use a different discretization (see section 6).

Next we decide the two members of the $k$ th base function, $B[k] . c$ and $B[k] . m$. Let $\left[L_{1}, L_{2}\right]$ be the support of the initial value $v_{0}(x)$ and $L_{1}=x_{n}<\cdots<x_{1}<$ $x_{0}=L_{2}$ be mesh points. Consider the self-similarity profiles that emanate from points $\left(x_{k}, v_{0}\left(x_{k}\right)\right)$. Then the center $c_{k}$ satisfies $g\left(\left(x_{k}-c_{k}\right) / t_{0}\right)=v_{0}\left(x_{k}\right)$. Taking the wave speed $f^{\prime}$ to both sides we get $c_{k}=x_{k}-t_{0} f^{\prime}\left(v_{0}\left(x_{k}\right)\right)$. We assign this center to $B[k] . c$, i.e.,

$$
B[k] . c=x_{k}-t_{0} f^{\prime}\left(v_{0}\left(x_{k}\right)\right) .
$$

Since $g\left(\left(\bar{x}-c_{1}\right) / t_{0}\right)=g\left(\left(\bar{x}-c_{2}\right) / t_{0}\right)$ at any point $\bar{x}>c_{1}, c_{2}$ implies $c_{1}=c_{2}$, we can easily see that two self-similarity profiles with the same time index never cross over to each other. So Lemma 4.1 implies that those centers are ordered by $L_{1}=B[n] . c<$ $\cdots<B[2] . c<B[1] . c$. Note that there is no self-similarity profile that emanates from the point $\left(x_{0}, v_{0}\left(x_{0}\right)\right)$.

The value of the second member $B[k] . m$ is given as the area enclosed by four (or three) curves, $y=v_{0}(x), y=0, y=g\left(\left(x-c_{k}\right) / t_{0}\right)$, and $y=g\left(\left(x-c_{k-1}\right) / t_{0}\right)$, i.e.,

$$
B[k] . m=\int_{x_{k}}^{x_{k-1}} v_{0}(x) d x+\int_{c_{k}}^{x_{k}} g\left(\frac{x-c_{k}}{t_{0}}\right) d x-\int_{c_{k-1}}^{x_{k-1}} g\left(\frac{x-c_{k-1}}{t_{0}}\right) d x,
$$

where $c_{0}=L_{2}$. Using relation (4.5), this is written in terms of the initial value and the flux:

$$
\begin{aligned}
B[k] . m=\int_{x_{k}}^{x_{k-1}} v_{0}(x) d x & +t_{0} v_{0}\left(x_{k}\right) f^{\prime}\left(v_{0}\left(x_{k}\right)\right)-t_{0} f\left(v_{0}\left(x_{k}\right)\right) \\
& -t_{0} v_{0}\left(x_{k-1}\right) f^{\prime}\left(v_{0}\left(x_{k-1}\right)\right)+t_{0} f\left(v_{0}\left(x_{k-1}\right)\right) .
\end{aligned}
$$

Consider the Cauchy problem (4.1) as an example. Since $f^{\prime \prime}(v)=1$ and $v_{x}(x, 0) \leq$ 1 , we may take any $t_{0}<1$. In the following examples we use $t_{0}=0.5$. In Figure $4.1(\mathrm{a})$, 10 self-similarity profiles are shown which emanate from 10 points $\left(j / 10, v_{0}(j / 10)\right)$, $j=0,1, \ldots, 9$. The centers are their $x$-intercepts.

In Figure 4.1(b), 10 base functions are displayed with initial value $v_{0}(x)$. Supports of these base functions are overlapped with each other. Their S-summation $u_{0}(x)=$ $\bigodot_{k=1}^{n} B[k]$ is considered as the initial discretization, which is the saw-tooth profile (solid lines) in Figure 4.1(a). Let $u_{0}^{\varepsilon}$ be such an approximation with a uniform mesh size $x_{k-1}-x_{k}=\varepsilon$. Then the sizes of the triangle-like areas in Figure 4.1(a), added to and subtracted from the area enclosed by $y=v_{0}(x)$ and the $x$-axis, are proportional to $\varepsilon^{2}$, and the total number of them has order $O(1 / \varepsilon)$. So we have $\left\|v_{0}-u_{0}^{\varepsilon}\right\|_{1}=O(\varepsilon)$ as $\varepsilon \rightarrow 0$, where $u_{0}^{\varepsilon}(x)=\bigodot_{k=1}^{n} B[k]$ with $t_{k}=t_{0}$ for all $k$. (Step 1 is complete.)

Theorem 3.6 implies that $u(x, t)=\bigodot_{k=1}^{n} B[k]$ with $t_{k}=t_{0}+t$ is the solution with the modified initial data $u_{0}$. So the rest of the scheme is focused on how to 


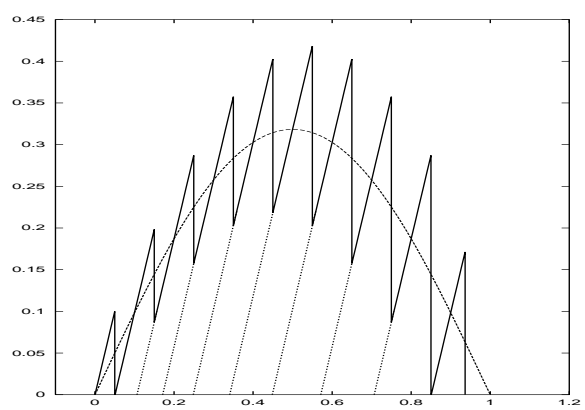

(a) The equal area rule

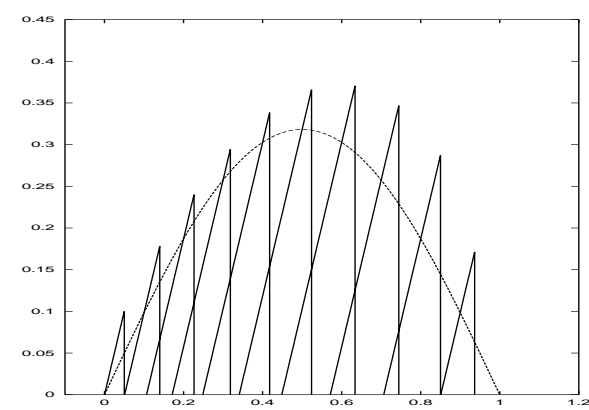

(b) Base functions with overlaps

FIG. 4.1. The initial value is approximated by a piecewise self-similar function, which is a saw-tooth profile in (a). It turns out to be an $S$-summation of base functions in (b).

display the given solution. Even if it is possible to follow the inductive arguments of the definition, we will get serious complexity in the coding if behind shocks capture the front ones, $\xi_{j-1}<\xi_{j}$, where $\xi_{j}$ is the shock location generated by the $j$ th base function (see the definition (3.13)-(3.15)). Since the S-summation is not changed even if these two base functions are merged before the summation (see Remark 3.5), we consider the merging process first.

Suppose that $\bigodot_{k=1}^{j-1} B[k]$ is achieved and $\xi_{j-1}<\xi_{j-2}<\cdots<\xi_{1}$. To obtain $\bigodot_{k=1}^{j} B[k]$ we need to check if $\xi_{j}<\xi_{j-1}$. Since $\xi_{j-1} \neq s_{j-1}$ in general, an equation corresponding to (3.18) does not provide the information we need. In the following we define an operator using a modified version of (3.18).

DEFINITION 4.2. We define a binary operator "*” between two base functions $B_{m_{k}, t_{k}, c_{k}, s_{k}}, k=1,2$, satisfying $c_{2}<c_{1}$. First, if $s_{2} \leq c_{1}$, we define $B_{m_{2}, t_{2}, c_{2}} *$ $B_{m_{1}, t_{1}, c_{1}} \equiv s_{2}$. If $c_{1}<s_{2}, B_{m_{2}, t_{2}, c_{2}} * B_{m_{1}, t_{1}, c_{1}}(\equiv \xi)$ is defined as the solution of

$$
F(\xi) \equiv \int_{c_{2}}^{\xi} g\left(\frac{x-c_{2}}{t_{2}}\right) d x-\int_{c_{1}}^{\xi} g\left(\frac{x-c_{1}}{t_{1}}\right) d x-m_{2}=0 .
$$

Let $\xi=B[j] * B[j-1]$. From (3.14) we can clearly see that $\xi=\xi_{j}$ if and only if $\xi \leq \xi_{j-1}$. If $\xi_{j-1}<\xi$, we also have $\xi_{j-1}<\xi_{j}$ and we may merge two base functions, $B[j]$ and $B[j-1]$, before the S-summation. On the other hand, since we have assumed $\xi_{j-1}<\xi_{j-2}<\cdots<\xi_{1}$, we have $\xi_{j-1}=B[j-1] * B[j-2]$. So we may conclude that

$$
\xi_{j}>\xi_{j-1} \quad \text { if and only if } B[j] * B[j-1]>B[j-1] * B[j-2] .
$$

So this operator gives the criterion for deciding if two base functions should be merged together or not. Furthermore, after the merging process, it gives the correct (artificial) shock locations $\xi_{j}(t)$ for the S-summation.

Step 2 (merging). In this step base functions are re-indexed for $k=1,2, \ldots, n^{\prime}$ whenever two base functions are merged together and the total number of base functions is decreased. Suppose that this merging procedure has been completed for all $k<j$ and $\xi_{j-1}<\cdots<\xi_{2}<\xi_{1}=s_{1}$ holds. Then $\xi_{k}=B[k] * B[k-1]$ for $k=2, \ldots, j-1$. Now we check the next base function $B[j]$.

If $B[j] * B[j-1]<B[j-1] * B[j-2]$, then $\xi_{j}=B[j] * B[j-1]$ and this step is completed for $k \leq j$. Suppose that $B[j] * B[j-1]>B[j-1] * B[j-2]$. Then (4.12) 
implies that $\xi_{j}>\xi_{j-1}$, and we may merge $B[j]$ and $B[j-1]$ (see Remark 3.5). Put

$$
B[j] . m=B[j] . m+B[j-1] . m,
$$

remove $B[j-1]$, and then rearrange the array $B[\cdot]$ from $k=1$ to $k=n^{\prime}-1$, where $n^{\prime}$ is the total number of base functions left after the previous step. Since the combined base function may take over another one again, we continue this process until we get $\xi_{j}<\xi_{j-1}$ or $j=1$, decreasing the index $j$ by 1 . We continue this procedure from $j=2$ to $j=n^{\prime}$. Note that there is no base function $B[0]$ and we use a convention $B[1] * B[0]:=B[1] . s$ in (4.12) for $j=2$, where $B[1] . s$ is the shock location of the base function given by the relation (3.3).

In Figure 4.2(a), 40 base functions $B_{m_{k}, c_{k}, t+t_{0}}, k=1, \ldots, 40$, are given at $t=1.5$ together with the exact solution we want to find. During the merging step, Step 2, 16 of them are merged together and a big base function emerges. The location and the size of the discontinuity of the newborn base function are almost identical to those of the physical shock. This big base function can be considered as an accumulation of small artificial shocks, and it represents the physical shock. (Step 2 is complete.)

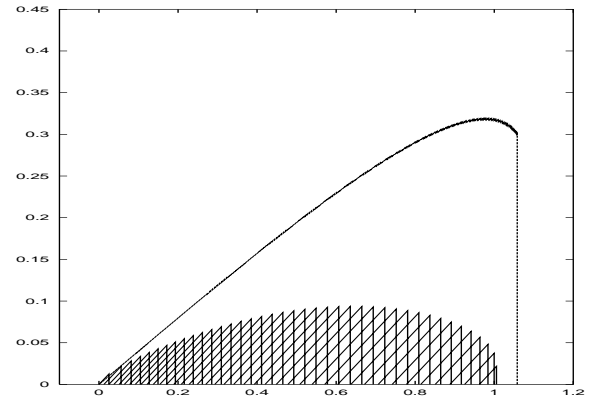

(a) 40 base functions before merging

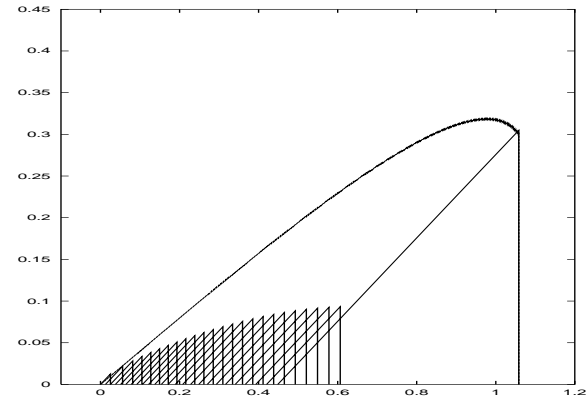

(b) 25 base functions left after merging

FIG. 4.2. 40 base functions have the slope $1 /\left(t_{0}+t\right)$ at time $t>0$, which is 0.5 in (a). After the merging process, Step 2, 16 of them are merged together and a big base function emerges in (b). The outside wave is the exact solution we want to approximate.

Remark 4.3. In the previous algorithm, we solve (4.11) instead of doing the time marching. This relation gives the correct location of artificial shocks if the merging step is completed. Using the relation (4.5), the function $F(\xi)$ in (4.11) is written as

$F(\xi) \equiv\left(\xi-c_{2}\right) g\left(\frac{\xi-c_{2}}{t_{2}}\right)-t_{2} f\left(g\left(\frac{\xi-c_{2}}{t_{2}}\right)\right)-\left(\xi-c_{1}\right) g\left(\frac{\xi-c_{1}}{t_{1}}\right)+t_{1} f\left(g\left(\frac{\xi-c_{1}}{t_{1}}\right)\right)-m_{2}$.

So we can simplify the integral equation (4.11). To find the zero of $F(\xi)$ we may use the bisection method. If $B[j] * B[j-1]>B[1] . s$, clearly we need to merge $B[j]$ and $B[j-1]$. So we may use $(B[j-1] . c, B[1] . s)$ as the initial interval.

If we use Newton's method, we need to study the structure of the self-similarity profile first. Let $t_{1}=t_{2}(\equiv t)$. The first two derivatives of $F(\xi)$ are

$$
F^{\prime}(\xi)=g\left(\frac{\xi-c_{2}}{t}\right)-g\left(\frac{\xi-c_{1}}{t}\right), \quad F^{\prime \prime}(\xi)=\frac{1}{t}\left(g^{\prime}\left(\frac{\xi-c_{2}}{t}\right)-g^{\prime}\left(\frac{\xi-c_{1}}{t}\right)\right) .
$$

Since the self-similarity profile $g(x)$ is an increasing function, we have $F^{\prime}(\xi)>0$ and (4.11) has a unique solution. On the other hand, since there is no monotonicity on $g^{\prime}(x)$ in general, we need to consider the structure $g(x)$ for the initial guess. 
Remark 4.4. With the power law $f(u)=u^{\gamma} / \gamma, u>0$, and the identical time index $t_{1}=t_{2} \equiv t,(4.11)$ is written as

$$
F(\xi)=\left(\xi-c_{2}\right)^{\frac{\gamma}{\gamma-1}}-\left(\xi-c_{1}\right)^{\frac{\gamma}{\gamma-1}}-\frac{\gamma}{\gamma-1} m_{2} t^{\frac{1}{\gamma-1}}=0 .
$$

For the Burgers case, $\gamma=2$, the operator is explicitly given by

$$
B_{m_{2}, t, c_{2}} * B_{m_{1}, t, c_{1}}=\frac{2 m_{2} t+c_{1}^{2}-c_{2}^{2}}{2\left(c_{1}-c_{2}\right)} .
$$

Remark 4.5. If there is no base function merged, there will be $n-1$ comparisons of (4.12). If $m$ base functions are merged, then $n-m$ base functions are left and the maximum number of comparisons (4.12) is $n+m-1<2 n$, which is of order $O(N)$.

Step 3 (displaying). Now we are ready to display the solution. Suppose that base functions $B[j], j=1, \ldots, n^{\prime}$, are left after the merging step. Let $\xi_{j}=B[j] * B[j-1]$. Then the right- and the left-hand side limits are

$$
\begin{gathered}
u\left(\xi_{j}+, T\right)=g\left(\left(\xi_{j}-B[j-1] . c\right) /\left(t+t_{0}\right)\right), \\
u\left(\xi_{j}-, T\right)=g\left(\left(\xi_{j}-B[j] . c\right) /\left(t+t_{0}\right)\right) .
\end{gathered}
$$

So to display the solution it is enough to plot the points $\left(\xi_{j}, u\left(\xi_{j}+, T\right)\right),\left(\xi_{j}, u\left(\xi_{j}-, T\right)\right)$ for $j=1, \ldots, n^{\prime}$. Between these points the solution has the self-similarity profile. So if we connect these points with the self-similarity profile with time index $t+t_{0}$ and center $B[j] . c$, we get the solution.

In Figure 4.3(b), the S-summation of the 25 base functions at time $t=1.5$ (see Figure $4.2(\mathrm{~b})$ ) has been displayed. We may observe that the exact solution passes through the artificial discontinuities of the approximation. Furthermore, we can clearly see that the initial error $\left\|v_{0}(x)-u_{0}(x)\right\|_{1}$ has been decreased a lot. (Step 3 is complete.)

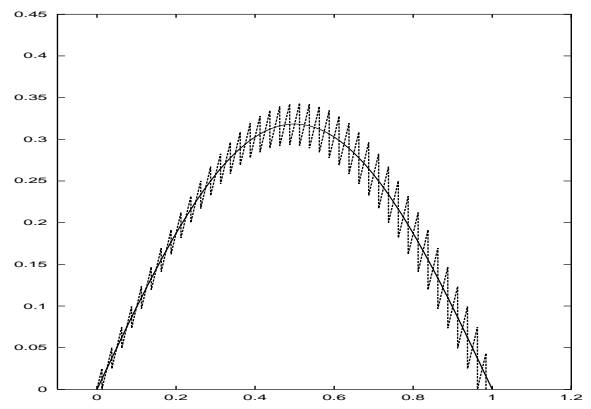

(a) Initial discretizations

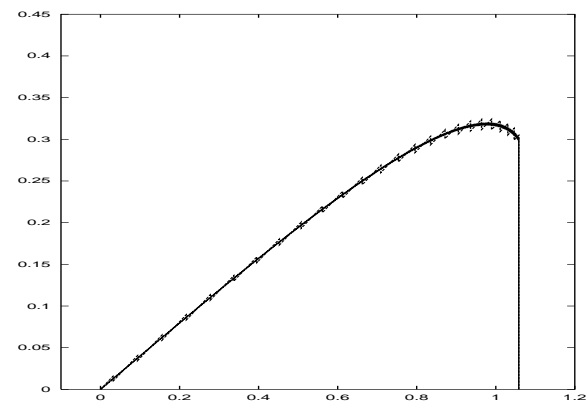

(b) Solutions at $t=1.5$

FIG. 4.3. The initial value of the problem (4.1) has been discretized using 40 base functions in (a). 25 base functions are left after the merging step with $t=1.5$, Figure $4.2(\mathrm{~b})$, and the $S$-summation gives the final approximation for the solution. We may observe that the exact solution, which has obtained using finer mesh points, passes through each of the artificial shocks.

Remark 4.6. One of the main features of the scheme introduced is that it has a complexity of order $O(N)$. Even though we have introduced extra complexities for solving (4.4) and (4.11), this does not increase the order of the complexity. On the other hand, for the convenience of the explanation, we have rearranged the whole array of base functions whenever one of them is merged to another. Since this rearranging 
TABLE 4.1

$C P U$ time comparison: Computations for (4.17) with $t_{0}=\pi / 2, t=4.0$. The number of base functions used initially is $N$, and $L$ of them are left after the merging step. CPU times for each step of the scheme are compared.

\begin{tabular}{rrrrrrr}
\hline$N$ & $L$ & Discretization & Merging & Displaying & Total & Order $\alpha$ \\
\hline 10 & 8 & 0 & 0 & 1 & 1 & \\
100 & 64 & 0 & 5 & 4 & 9 & 0.954 \\
1000 & 628 & 0 & 57 & 34 & 91 & 1.005 \\
10000 & 6272 & 2 & 617 & 357 & 976 & 1.030 \\
100000 & 62707 & 22 & 7763 & 3956 & 11741 & 1.080 \\
\hline
\end{tabular}

process will increase the order of the complexity, we need to use a different strategy in the actual computation. We may link the base functions pointing the adjacent ones so that one of them can be easily removed. These kinds of techniques are classical and we omit the details.

4.3. CPU time comparison. In this section we consider several numerical examples and show the CPU time for each case. In fact, the Burgers equation is the case that the self-similarity profile $g(x)$ and the binary operator " $*$ " between base functions are given explicitly, (4.3) and (4.15). So that case does not show the complexity of the scheme well. In the examples in this section, we numerically solve (4.4) and (4.11) using Newton's method.

First, consider a Cauchy problem with the cubic law, $f(v)=v^{3} / 3$,

$$
\begin{gathered}
v_{t}+v^{2} v_{x}=0, \\
v(x, 0)=v_{0}(x),
\end{gathered} v_{0}(x)=\left\{\begin{array}{cl}
\sin (\pi x) / \pi, & 0<x<1, \\
0, & \text { otherwise. }
\end{array}\right.
$$

In this case the self-similarity profile $g(x)$ is concave. Since $v_{0}^{\prime}(x)=\cos (\pi x)$ and $f^{\prime \prime}(v)=2 v$, the condition (4.6) is written as

$$
\cos (\pi x)<\frac{\pi}{2 t_{0} \sin (\pi x)}, \quad 0<x<1 .
$$

We can easily check that it is satisfied for $t_{0}=\pi / 2$. In Table 4.1 we have compared the CPU time of the computations as we increase the number of base functions (or mesh points). The solution is computed for time $t=4$.

Suppose that the CPU time $T(N)$ for the computation with $N$ mesh points is $T(N)=c N^{\alpha}$ for some constants $c, \alpha>0$. Then we can easily check that

$$
\alpha=\frac{\ln \left(T\left(N_{1}\right) / T\left(N_{2}\right)\right)}{\ln \left(N_{1} / N_{2}\right)} .
$$

This number represents the complexity order of the scheme, and it is computed and shown in Table 4.1. We may observe that the order is about $\alpha=1.08$. These computational results confirm that the complexity of the scheme is almost linear. The extra growth in the CPU time is caused by Newton's method. If we use finer base functions, we need to use smaller tolerances in finding the shock location.

Next we consider a problem with the flux $f(u)=\frac{2}{3} u^{3 / 2}$, where the self-similarity profile $g(x)$ is convex. In this case we cannot find the time index $t_{0}>0$ that satisfies (4.6) for the initial value given in the previous example. So we consider

$$
\begin{aligned}
& v_{t}+\sqrt{v} v_{x}=0, \\
& v(x, 0)=v_{0}(x),
\end{aligned} v_{0}(x)=\left\{\begin{array}{cl}
5 x^{2}(x-1)^{2}, & 0<x<1, \\
0, & \text { otherwise }
\end{array}\right.
$$


TABLE 4.2

CPU time comparison: Computations for (4.19) with $t_{0}=0.1, t=1.0$. The number of base functions used initially is $N$, and $L$ of them are left after the merging step. CPU times for each step of the scheme are compared.

\begin{tabular}{rrrrrrr}
\hline$N$ & $L$ & Discretization & Merging & Displaying & Total & Order $\alpha$ \\
\hline 10 & 7 & 0 & 1 & 1 & 2 & \\
100 & 62 & 0 & 13 & 7 & 20 & 1.000 \\
1000 & 610 & 0 & 209 & 92 & 301 & 1.178 \\
10000 & 6092 & 1 & 2785 & 1113 & 3899 & 1.112 \\
100000 & 60914 & 12 & 34068 & 13105 & 47185 & 1.083 \\
\hline
\end{tabular}

TABLE 4.3

CPU time comparison: Computations for (4.20) with $t_{0}=0.1, t=0.5$. The number of base functions used initially is $N$, and $L$ of them are left after the merging step. CPU times for each step of the scheme are compared.

\begin{tabular}{rrrrrrr}
\hline$N$ & $L$ & Discretization & Merging & Displaying & Total & Order $\alpha$ \\
\hline 10 & 7 & 0 & 1 & 0 & 1 & \\
100 & 60 & 0 & 7 & 5 & 12 & 1.079 \\
1000 & 589 & 1 & 113 & 53 & 167 & 1.144 \\
10000 & 5878 & 1 & 1635 & 633 & 2269 & 1.133 \\
100000 & 58767 & 13 & 19965 & 7114 & 27092 & 1.077 \\
\hline
\end{tabular}

Since $v_{0}^{\prime}(x)=10 x(x-1)(2 x-1)$ and $f^{\prime \prime}(v)=1 / 2 \sqrt{v}$ in this case, the condition (4.6) is written as

$$
-10(2 x-1)<2 / t_{0}, \quad 0<x<1 .
$$

It is satisfied for $t_{0}<0.2$, and we choose $t_{0}=0.1$. The solution has been computed at time $t=1$, and their CPU times and the complexity of the scheme have been compared in Table 4.2. We observe a similar complexity order, $\alpha=1.083$, as we do in the previous example.

As the last example, we consider a combination of three power laws,

$$
v_{t}+\left(\sqrt{v}+v+v^{2}\right) v_{x}=0, \quad v_{0}(x)=\left\{\begin{array}{cc}
5 x^{2}(x-1)^{2}, & 0<x<1 . \\
0(x, 0)=v_{0}(x), & \text { otherwise }
\end{array}\right.
$$

The solution has been computed at time $t=0.5$ using an initial time index $t_{0}=0.1$. Their CPU times and the complexity of the scheme have been compared in Table 4.3. We observe a complexity order $\alpha=1.077$ which is similar to the previous examples.

4.4. Comparison with Godunov. A typical way to discretize the initial data is to take the cell average (see Figure 4.4(a)). The Godunov scheme solves a series of Riemann problems between each cell for a short amount of time $\Delta t$ and then repeats the process until it reaches a given time $t>0$. In Figure 4.4(b) we can see that the numerical solution converges to the same limit as the S-summation shown in Figure 4.3(b), as $\Delta x \rightarrow 0$.

Remark 4.7 (computation time). Let $N$ be the number of mesh points. Then the number of operations for the S-summation is of order $O(N)$ since the time marching process is not required, Theorem 3.6. The number of operations is almost independent from the final time $t>0$. On the other hand, the Godunov scheme has operations of order $O\left(N^{2}\right)$ and the situation becomes worse if the final time $t$ is increased.

Remark 4.8 (error estimate). We can clearly observe that the exact solution $v$ of (4.1) (or $\|\mathcal{C}\| \rightarrow 0$ limit of the S-summation) passes through artificial shocks of 


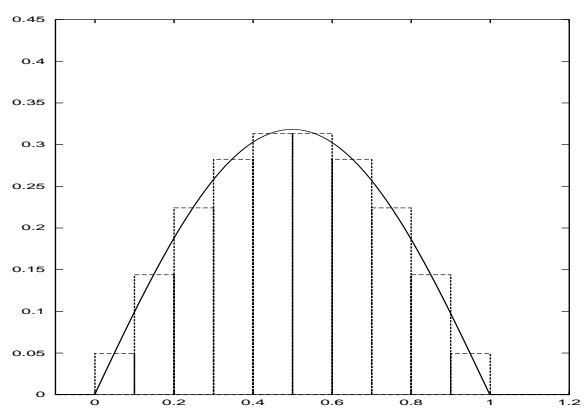

(a) Data discretization



(b) Solutions at $t=1.5$

FIG. 4.4. Three approximations by Godunov using $\Delta x=1 / 10,1 / 40,1 / 160$. The scheme is convergent to the same limit of the $S$-summation. We can observe that numerical solutions are separated near the shock, and it is hard to guess where the limit is from a single computation.

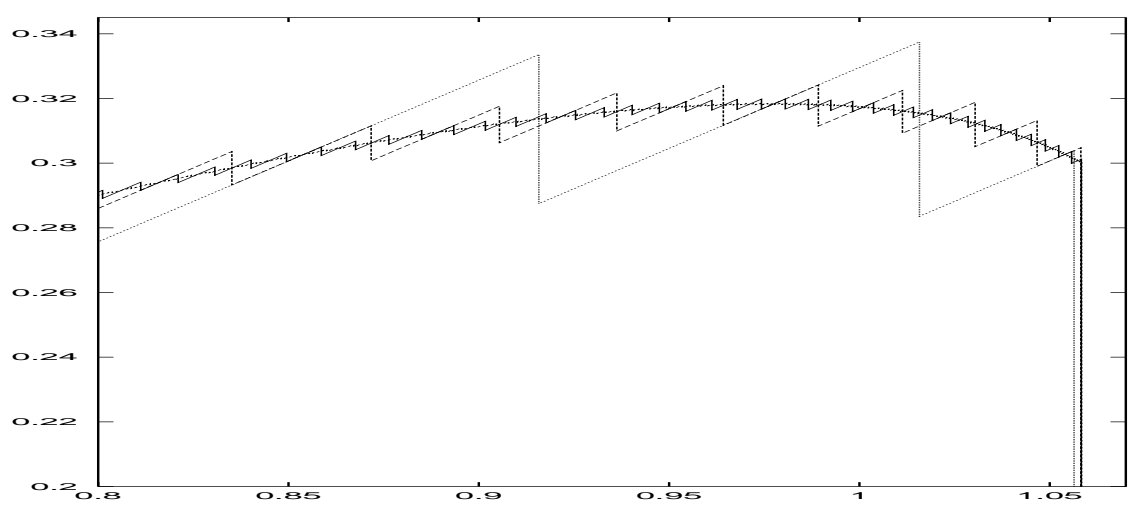

FIG. 4.5. A magnification of Figure 4.3(b) near the physical shock shows that an S-summation with a finer mesh passes through the middle of the artificial shocks. 10, 40, and 160 base functions are used.

self-similarity solutions (see Figure 4.3(b)). In Figure 4.5 a part of Figure 4.3(b) near the physical shock is magnified together with other similarity summations consisting of 10 and 160 base functions. In this figure we can also observe that S-summations are attached to each other in the middle of self-similar profiles. Noting that the sizes of artificial shocks decrease in time with order of $O\left(1 /\left(t+t_{0}\right)\right)$, these observations show the possibility for a good error estimate.

Remark 4.9 (shock appearance time). In a numerical scheme the solution is approximated by piecewise continuous functions, and it is hard to see if a discontinuity represents the physical shock or not. In our scheme, as we can see from Figure 4.2, the accumulation of base functions represents the physical shock. So, if a base function is merged to its behind one in the sense of (4.12), we may conclude that a physical shock has appeared. The physical shock appears at time $t=1$ in the example (4.1) since $\min \left(\partial_{x} v_{0}(x)\right)=-1$. We can easily check whether (4.12) happens around that time. Table 4.4 shows the time when the number of initial base functions decreases by one. 
TABLE 4.4

Shock appearance time. The exact solution with initial data (4.1) blows up at $t=1$. The time of shock appearance can be measured by counting the base functions after the merging step.

\begin{tabular}{cc}
\hline Initial number of base functions & The time when the number is decreased by 1 \\
\hline 25 & $T=1.02$ \\
50 & $T=1.005$ \\
100 & $T=1.0015$ \\
200 & $T=1.0008$ \\
400 & $T=1.0002$ \\
800 & $T=1.00005$ \\
\hline
\end{tabular}

5. General cases. The S-summation has been considered under hypothesis $(\mathrm{H})$. In this section we generalize it under hypotheses (H1) and (H2).

5.1. General convex flux. We consider $L^{1}$ initial function $u_{0}$ which is uniformly bounded, say, $-A \leq u_{0}(x) \leq B$. Then the solution of $(2.1)$ is bounded above and below:

$$
-A \leq u(x, t) \leq B, \quad A, B \in \boldsymbol{R}^{+} .
$$

Consider a general convex flux, i.e.,

$$
f^{\prime \prime}(u) \geq 0 .
$$

If the flux satisfies $f^{\prime \prime}(u) \leq 0$, we may change the variable $y=-x$ and get an equation $u_{t}+\bar{f}(u)_{y}=0$ with $\bar{f}(u)=-f(u)$, where $\bar{f}$ satisfies (H1). Note that we include the equality in (H1) and a piecewise linear flux can be considered.

We can easily check that a new flux,

$$
h(w)=f(w-A)-f^{\prime}(-A) w-f(-A),
$$

satisfies the hypothesis $(\mathrm{H})$ and $h^{\prime}(0)=0$. Let $w(x, t)$ be the solution of

$$
w_{t}+h(w)_{x}=0, \quad w(x, 0)=u_{0}(x)+A .
$$

We can easily check that

$$
u(x, t)=w\left(x-f^{\prime}(-A) t, t\right)-A
$$

is the solution with the original flux $f$ and initial data $u_{0}$. Since $u \geq-A$, the solution $w(x, t)$ is positive. Now we are in the exact same situation as in the previous sections, except with respect to the structure of the initial data. The initial data $w(\cdot, 0)$ is not $L^{1}$ anymore. To handle the situation, we consider two special base functions with infinite mass,

$$
\begin{gathered}
B_{t, c=-\infty, s}(x)=\left\{\begin{array}{cc}
A, & x<s+t h(A) / A, \\
0, & x>s+t h(A) / A,
\end{array}\right. \\
B_{t, c, s=\infty}(x)=\left\{\begin{array}{cc}
\max \left(g\left(\frac{x-c}{t}\right), A\right), & x>c, \\
0, & x<c,
\end{array}\right.
\end{gathered}
$$

where $h^{\prime}(g(x))=x$, i.e., $g(x)$ is the similarity profile under the flux $h(w)$, not $f(u)$. These base functions handle the transformation $u_{0}(x) \rightarrow u_{0}(x)+A$. Note that the speed of the shock connecting the state $w=A$ and $w=0$ is $h(A) / A$ in our case. The S-summation including these two base functions can be defined in a similar way. We omit the details. Figure 5.1 shows how the self-similar solution evolves for the Burgers case. In the figure even the solution with very rough initial discretization with only 16 base functions represents the asymptotic behavior very correctly. 


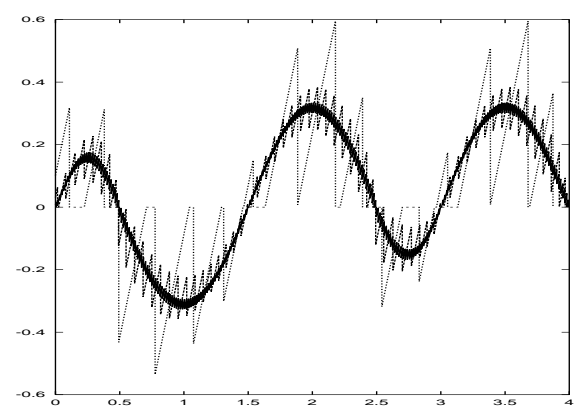

(a) Data discretization

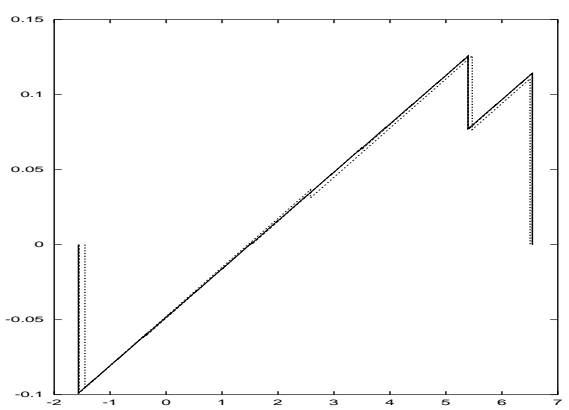

(b) Solutions at $t=30$

FIG. 5.1. Three S-summations are displayed using 16, 64, and 256 base functions. It handles sign changing solutions correctly. This figure shows the time convergence to an inviscid $N$-wave.

5.2. Flux without convexity. Consider a flux with a single inflection point:

$$
f^{\prime \prime}(u) \leq 0 \text { for } u \leq A, \quad f^{\prime \prime}(u) \geq 0 \text { for } u \geq A
$$

Then, under the change of variables,

$$
h(w)=f(w+A)-f^{\prime}(A) w-f(A), \quad u(x, t)=w\left(x-f^{\prime}(A) t, t\right)+A,
$$

the problem (2.1) is transformed to

$$
w_{t}+h(w)_{x}=0, \quad w(x, 0)=u_{0}(x)-A .
$$

Then the new flux $h$ satisfies

$$
h^{\prime \prime}(w) \leq 0 \text { for } w \leq 0, \quad h^{\prime \prime}(w) \geq 0 \text { for } w \geq 0, \quad h^{\prime}(w) \geq 0 \text { for all } w,
$$

and $h(0)=h^{\prime}(0)=h^{\prime \prime}(0)=0$. Since $A$ is not the lower bound of the solution $u(x, t)$ in general, we cannot expect $w \geq 0$. So in this case we have to consider the positive part and the negative part together. It is possible since $h^{\prime}(w)$ is monotone on $(-\infty, 0)$ and $(0, \infty)$, respectively. All we have to do is to consider negative base functions together with positive ones. Since the wave speed $h^{\prime}(w)$ is positive, the S-summation is defined from the right-hand side as in the previous cases.

Example 5.1. Consider an inviscid thin film flow in [1],

$$
\begin{gathered}
u_{t}+\left(u^{2}-u^{3}\right)_{x}=0, \\
u(x, 0)=u_{0}(x),
\end{gathered}
$$

where the initial datum is compactly supported $\operatorname{supp}\left(u_{0}\right) \subset\left[L_{1}, L_{2}\right]$. The flux $f(u)=$ $u^{2}-u^{3}$ has a single inflection point $A=1 / 3$ and, under the transformation (5.4), we get the flux $h(w)=-w^{3}$. It satisfies

$$
h^{\prime \prime}(w) \geq 0 \text { for } w \leq 0, \quad h^{\prime \prime}(w) \leq 0 \text { for } w \geq 0, \quad h^{\prime}(w) \leq 0 \text { for all } w,
$$

which is not exactly the same as (5.5) but has the opposite direction in the inequalities. We may do the S-summation from the left-hand side instead of changing the space variable using $y=-x$. Now the original problem (5.6) is transformed to

$$
\begin{gathered}
w_{t}-\left(w^{3}\right)_{x}=0 \\
w(x, 0)=w_{0}(x):=u_{0}(x)-A
\end{gathered}
$$


In this case the self-similarity profile (2.8) is given by

$$
g_{ \pm}(x)= \pm \sqrt{-x / 3}, \quad x<0,
$$

and the corresponding base functions are

$$
B_{t, s, c}^{ \pm}(x)=\left\{\begin{array}{cl}
g_{ \pm}((x-c) / t), & s<x<c \\
0, & \text { otherwise }
\end{array}\right.
$$

The initial data $v_{0}(x)$ converges to $-A$ as $x \rightarrow \pm \infty$, and we need to consider two base functions with infinite mass that are

$$
\begin{gathered}
B_{t, s=L_{2}, c=\infty}(x)=\left\{\begin{array}{cc}
-A, & x>L_{2}+t h(-A) /(-A), \\
0, & x<L_{2}+t h(-A) /(-A),
\end{array}\right. \\
B_{t, s=-\infty, c=L_{1}}(x)=\left\{\begin{array}{cc}
\max \left(g_{-}\left(\left(x-L_{1}\right) / t\right),-A\right), & x<L_{1}, \\
0, & x>L_{1} .
\end{array}\right.
\end{gathered}
$$

Note again that in our example (5.6) the infinite state is $-A=-1 / 3$ and the shock speed is $h(-A) /(-A)=-1 / 9$.

Numerical solutions of (5.6) with initial data,

$$
u_{0}(x)=\left\{\begin{array}{cl}
\frac{2}{3}|\sin (2 \pi x)|, & 0<x<2, \\
0, & \text { otherwise }
\end{array}\right.
$$

are shown in Figure 5.2. The first picture shows the initial data and the S-summation of 200 base functions at time $t=6$. A part of the summation has been magnified with numerical approximations of the Godunov scheme in the second picture. We can clearly see that the solution of the Godunov scheme converges to the S-summation. This example shows that the S-summation gives a very accurate resolution using a small number of mesh points.

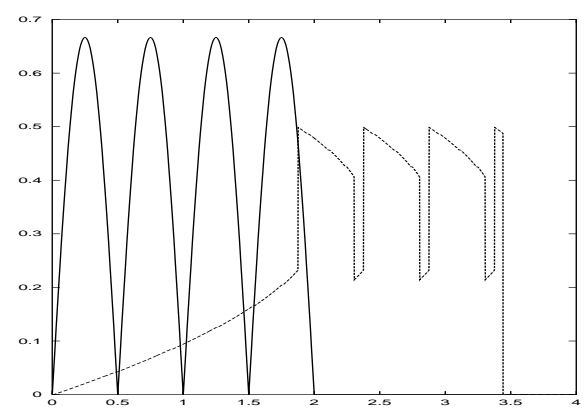

(a) Initial data and $S$-summation at $t=6$

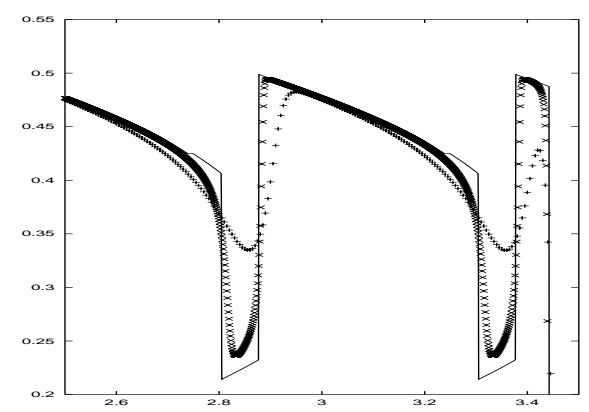

(b) Comparison with Godunov

FIG. 5.2. Flux is $f(u)=u^{2}-u^{3}$. (a) shows the initial data and the $S$-summation at $t=6$. (b) shows that the Godunov scheme converges to the $S$-summation. 200 base functions are used in the S-summation and 800 and 4,000 meshes are used in the Godunov scheme. 
5.3. Space dependent flux in multidimensional space. The self-similarity of the problem (2.1) relies on the fact that the flux depends only on the solution, i.e., $f=f(u)$. We have no clue how to generalize our scheme to a problem with a general space dependent flux, $f=f(u, x)$. However, if the space dependence is given by

$$
u_{t}+a(x) f^{\prime}(u) u_{x}=0
$$

the equation is transformed into

$$
u_{t}+f(u)_{y}=0
$$

under the change of variable $y(x)=\int_{0}^{x} 1 / a(s) d s$, and our scheme can be applied.

Since the self-similarity of hyperbolic conservation laws is the one-dimensional property, it should be possible to expand the scheme to multidimensional problems. Consider a two-dimensional problem,

$$
u_{t}+f^{\prime}(u)\left(a\left(x_{1}, x_{2}\right) u_{x_{1}}+b\left(x_{1}, x_{2}\right) u_{x_{2}}\right)=0,
$$

with a velocity vector field satisfying

$$
\partial_{x_{1}} a\left(x_{1}, x_{2}\right)+\partial_{x_{2}} b\left(x_{1}, x_{2}\right)=0 .
$$

Cvetkovic and Dagans [6] suggest space variables $y_{1}, y_{2}$ satisfying

$$
\frac{d y_{1}}{d x_{1}}=\frac{1}{a\left(x_{1}, \eta\right)}, \quad y_{2}=x_{2}-\eta, \quad \frac{d \eta}{d x_{1}}=\frac{b\left(x_{1}, \eta\right)}{a\left(x_{1}, \eta\right)},
$$

which transform (5.14) into

$$
u_{t}+f(u)_{y_{1}}=0, \quad u=u\left(y_{1}, y_{2}, t\right) .
$$

Problem (5.16) can be considered as a set of one-dimensional problems, and, hence, the complexity of the scheme for it is of order $O\left(N^{2}\right)$. Since the transformation (5.15) also has the complexity of $O\left(N^{2}\right)$, we eventually get a scheme of $O\left(N^{2}\right)$ for a twodimensional problem. In this approach, each channel of the velocity vector field is considered separately and, hence, it seems useful to channel problems.

6. Second order approximation. The scheme introduced in the previous sections exactly solves the problem with modified initial data, and the size of the initial error decreases in time. However, the scheme is not good enough for the short time behavior since the error generated by the initial discretization can be huge. Here we add an extra structure to base functions and make the initial data discretization to be of second order. In this way we can handle general piecewise self-similar solutions in (1.7).

6.1. Modified base functions. The base function considered in the previous sections has three indexes, say, $m, t, c$. In this section we introduce two extra indexes, $h$ and $\bar{t}$. Note that there are two time indexes $t$ and $\bar{t}$ which play different roles. We assume $0 \leq t<\infty$ and $-\infty<\bar{t} \leq \infty$. For simplicity we assume (H). It can be easily generalized, as it was in section 5 .

To figure out the structure of the new base function $B_{m, t, c}^{h, \bar{t}}(x)$, we introduce

$$
x^{*}=c+t f^{\prime}(h), \quad 0 \leq t<\infty,
$$


and

$$
\bar{c}=x^{*}-\bar{t} f^{\prime}(h), \quad-\infty<\bar{t}<\infty,
$$

(see Figures 6.1 and 6.2 ). Let $g(x)$ be the self-similarity profile. As an intermediate step we define $B_{t, c}^{h, \bar{t}}(x)$ first. For $0<\bar{t}<\infty$ it is defined by

$$
B_{t, c}^{h, \bar{t}}(x)=\left\{\begin{array}{cl}
g((x-c) / t), & c<x<x^{*} \\
g((x-\bar{c}) / \bar{t}), & x^{*}<x \\
0, & \text { otherwise }
\end{array}\right.
$$

and, for $-\infty<\bar{t} \leq 0$, it is defined by

$$
B_{t, c}^{h, \bar{t}}(x)=\left\{\begin{array}{cl}
g((x-c) / t), & c<x<x^{*} \\
g((x-\bar{c}) / \bar{t}), & x^{*}<x<\bar{c} \\
0, & \text { otherwise }
\end{array}\right.
$$

The constant $\bar{c}$ is the center of the top self-similarity profile with time index $\bar{t}$, and the constant $x^{*}$ is the $x$-coordinate of the intersection point between two selfsimilarity profiles with indexes $t$ and $\bar{t}$ (see Figures 6.1 and 6.2). We can easily see from (6.2) that $\bar{c}<x^{*}$ for $\bar{t}>0$ and $\bar{c}>x^{*}$ for $\bar{t}<0$. The function $B_{t, c}^{h, \bar{t}}(x)$ is well defined for $t=0, \bar{t}=0$ since the corresponding domain is empty. For $\bar{t}=\infty$, we consider

$$
B_{t, c}^{h, \infty}(x)=\left\{\begin{array}{cl}
g((x-c) / t), & c<x<x^{*} \\
h, & x^{*}<x \\
0, & \text { otherwise }
\end{array}\right.
$$

Now we introduce the index $m>0$, which decides the support of the base function. Let $\xi>c$ be the solution of

$$
\int_{c}^{\xi} B_{t, c}^{h, \bar{t}}(x) d x=m
$$

For $\bar{t}>0$ it always has a solution. For $\bar{t} \leq 0$ it has a solution only if $m<\int_{c}^{\bar{c}} B_{t, c}^{h, \bar{t}}(x) d x$. The base function is now defined by

$$
B_{m, t, c}^{h, \bar{t}}(x)=\left\{\begin{array}{cl}
B_{t, c}^{h, \bar{t}}(x), & c<x<\xi \\
0, & \text { otherwise }
\end{array}\right.
$$

Let $u(x, t)$ be the solution of the conservation law $u_{t}+f(u)_{x}=0$ with its initial value $u(x, 0)=B_{m, 0, c}^{h, \bar{t}}(x)$. Then, from the well-known technique of equal area construction, we may easily see that the solution is simply $u(x, t)=B_{m, t, c}^{h, \bar{t}+t}(x)$ (see Figures 6.1 and 6.2). For this solution $u(x, t)$, the point $x^{*}=x^{*}(t)$ in (6.1) satisfies

$$
x^{*}(t)=c+t f^{\prime}(h)=c+t f^{\prime}\left(u\left(x^{*}(t), t\right)\right), \quad x^{*}(t)<\xi(t),
$$

where $\xi=\xi(t)$ is the solution of (6.6). So $x=x^{*}(t)$ is a characteristic line for $x^{*}(t)<\xi(t)$. On the other hand, $\bar{c}$ is a constant with respect to $t>0$ :

$$
\bar{c}=x^{*}(t)-(t+\bar{t}) f^{\prime}(h)=c-\bar{t} f^{\prime}(h) .
$$




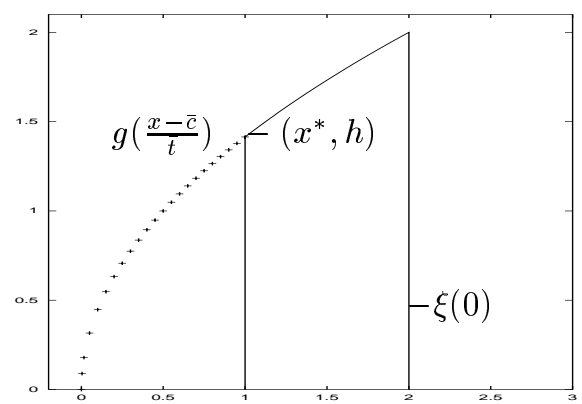

(a) Base function $B_{m, 0,1}^{h, \bar{t}}(x), \bar{t}>0$

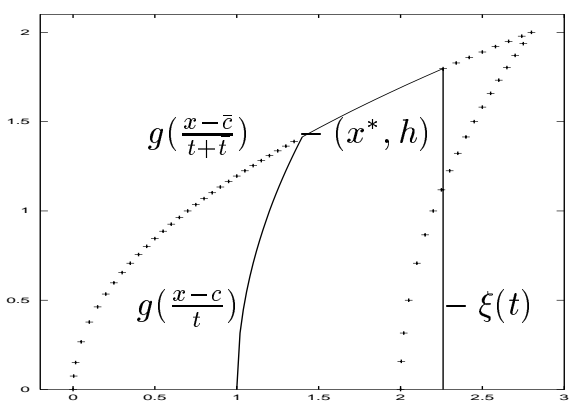

(b) Base function $B_{m, t, 1}^{h, t+\bar{t}}(x)$

FIG. 6.1. If the flux is $f(v)=v^{3} / 3$, the self-similarity profile is $g(x)=\sqrt{x}$. Base function $B_{m, 0, c}^{h, \bar{t}}(x)$ with $c=1, \bar{t}=0.5, h=\sqrt{2}, m=\int_{1}^{2} g(x / 0.5) d x$ is given in (a) (solid lines). We can easily check that $\bar{c}=0$. If $u(x, 0)=B_{m, 0, c}^{h, \bar{t}}(x)$, the solution of the conservation law is $u(x, t)=B_{m, t, c}^{h, t+\bar{t}}(x)$ and it is given in (b) (solid lines) with $t=0.2$.

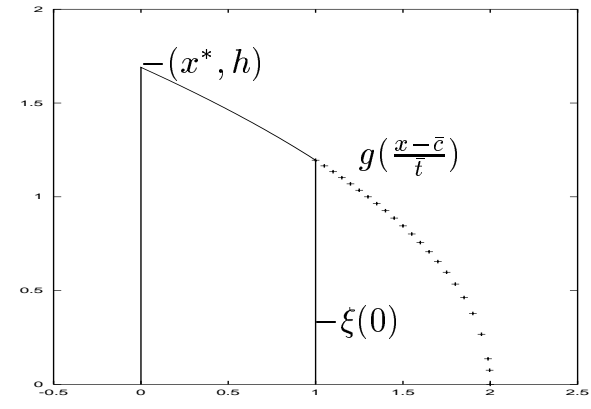

(a) Base function $B_{m, 0,0}^{h, \bar{t}}(x), \bar{t}<0$

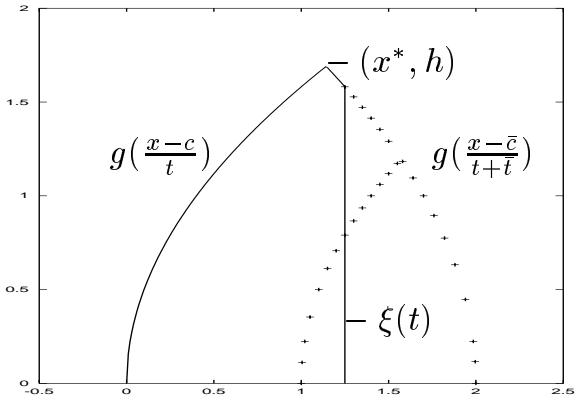

(b) Base function $B_{m, t, 0}^{h, t+\bar{t}}(x)$

FIG. 6.2. If the flux is $f(v)=v^{3} / 3$, the self-similarity profile is $g(x)=\sqrt{x}$. Base function $B_{m, 0, c}^{h, \bar{t}}(x)$ with $c=0, \bar{t}=-0.7, h=\sqrt{2 / 0.7}, m=\int_{0}^{1} g(2-x / 0.7) d x$ is given in (a) (solid lines). We can easily check that $\bar{c}=2$. If $u(x, 0)=B_{m, 0, c}^{h, \bar{t}}(x)$, the solution of the conservation law is $u(x, t)=B_{m, t, c}^{h, t+\bar{t}}(x)$ and it is given in (b) (solid lines) with $t=0.4$.

In Figures 6.1 and 6.2 base functions are displayed for positive and negative $\bar{t}$ together with self-similarity profiles. In these figures we can clearly observe the different roles of two self-similarity profiles generated by two index sets $\{c, t\}$ and $\{\bar{c}, \bar{t}\}$.

The S-summation among these base functions can be similarly defined using the profile $g((x-c) / t)$ in the domain $c<x<x^{*}$ and the profile $g((x-\bar{c}) / \bar{t})$ for $x^{*}<x$. We omit the details. We may consider the base function (3.2) as a special case of (6.7) with $\bar{t}=0$.

6.2. Initial discretization and the approximation. Suppose the initial function $v_{0} \in L^{1}$ has a compact $\operatorname{support} \operatorname{supp}\left(v_{0}\right) \subset\left[L_{1}, L_{2}\right]$. Let $\mathcal{C}=\left\{c_{n}=L_{1}<\cdots<\right.$ $\left.c_{1}<c_{0}=L_{2}\right\}$ be a partition of the interval $\left[L_{1}, L_{2}\right]$. We can approximate $v_{0}$ with self-similarity profiles over interval $\left(c_{k}, c_{k-1}\right)$ with time index $\bar{t}_{k} \in \boldsymbol{R}$, which is second order. For the Burgers case it is simply a piecewise linear approximation. The 
approximation $u_{0}$ can be written as

$$
u_{0}(x)=\bigodot_{k=1}^{n} B_{m_{k}, 0, c_{k}}^{h_{k}, \bar{t}_{k}}(x)=\sum_{k=1}^{n} B_{m_{k}, 0, c_{k}}^{h_{k}, \bar{t}_{k}}(x),
$$

where $m_{k}=\int_{c_{k}}^{c_{k-1}} u_{0}(x) d x$ and $h_{k}=u_{0}\left(c_{k}\right)$. Initially, the supports of base functions are disjoint, and, hence, the self-similarity summation is the usual summation. The exact solution $v(x, t)$ of the conservation law (1.1) is approximated by

$$
u(x, t)=\bigodot_{k=1}^{n} B_{m_{k}, t, c_{k}}^{h_{k}, t+\bar{t}_{k}}(x),
$$

and we expect an error estimate similar to (3.16), i.e.,

$$
\|v(x, t)-u(x, t)\|_{1} \leq\left\|v_{0}(x)-u_{0}(x)\right\|_{1}=O\left(\|\mathcal{C}\|^{2}\right) \quad \text { as } \quad\|\mathcal{C}\| \rightarrow 0 .
$$

Remark 6.1. The initial discretization (6.8) is trivial in comparison with Step 1 in section 4.2. It is an additional advantage we obtain when the modified base function is used in a numerical scheme. However, this additional structure may cause extra complexity when it is used as an analytical tool.

Remark 6.2 (piecewise constant data). In many cases initial data are given as piecewise constant functions from the beginning. In this case the initial data can be considered as a summation of base functions with $\bar{t}=\infty$; see (6.5). In Figure 6.3 we consider the Burgers case (4.1) using base functions $B_{m, t, c}^{h, \infty}(x)$. We can clearly see that these approximations represent the shock location very well. Unlike the previous case, the solution with finer mesh always passes though the constant parts of coarse ones.

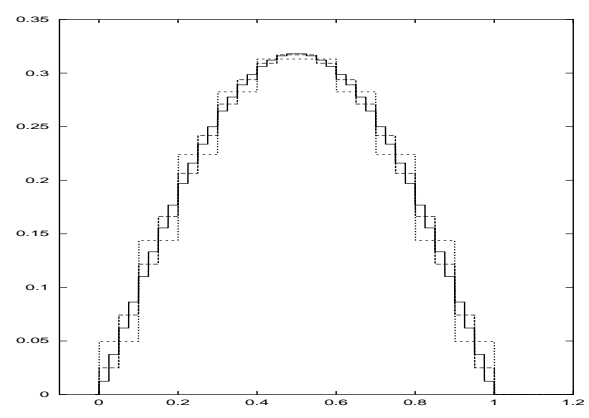

(a) Data discretization

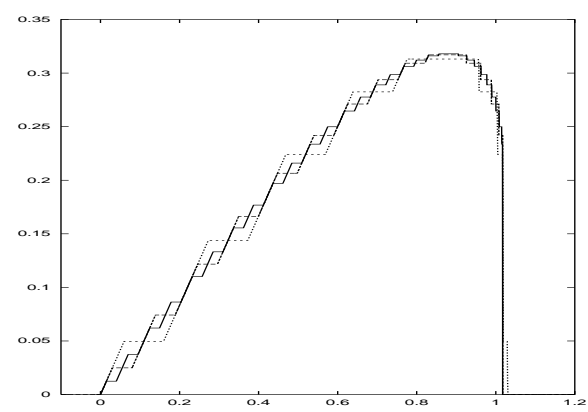

(b) Solutions at $t=1.2$

FIG. 6.3. The S-summation for the modified base functions (6.7) with $\bar{t}=\infty$ gives a piecewise constant, piecewise self-similar solution. In the figure, 3 summations are displayed together using 10, 20, 40 base functions. We observe that the finer one always passes the constant parts.

Remark 6.3 (singular initial data). If singular initial data are given, then extra mesh points are usually introduced to capture the effect of the singularity of the data. But since our method handles initial data individually, extra mesh points are not needed. In Figure 6.4(a) the Burgers equation is solved with singular initial data. We use 6 modified base functions with $\bar{t}=\infty$.

Remark 6.4 (front tracking). It is possible to consider the front tracking method in terms of the S-summation. Consider an $L^{1}$ solution of the Burgers equation 


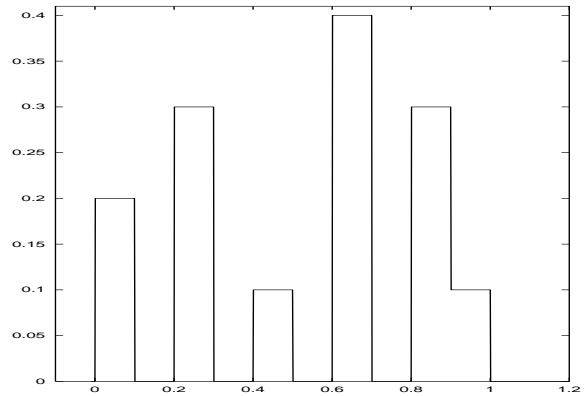

(a) Singular initial data

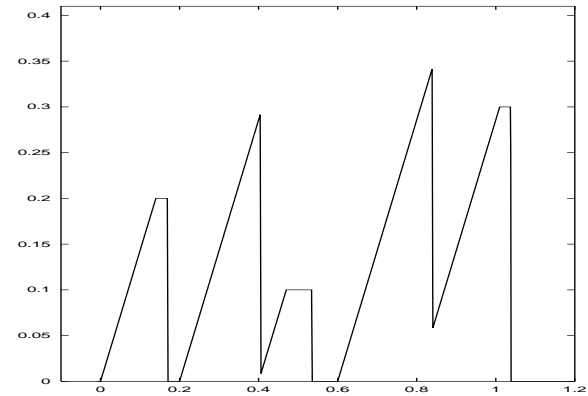

(b) Solutions at $t=0.7$

FIG. 6.4. The scheme does not require extra meshes to handle singular initial data (a). In the $S$-summation every datum is handled exactly by a base function. Only 6 base functions solve this example.

bounded by $0 \leq u(x, t) \leq 1$. Let $h(u)$ be the polygonal approximation of the flux $f(u)=u^{2} / 2$ with the partition $\{0,1 / n, \ldots, n / n=1\}$. Then $h^{\prime}(u)$ is a step function,

$$
h^{\prime}(u)=(2 k-1) / 2 n, \quad(k-1) / n<u<k / n, \quad k=1, \ldots, n,
$$

and the self-similarity profile $g(x)$ is given by

$$
g(x)=(k-1) / n, \quad(2 k-1) / 2 n<x<(2 k+1) / 2 n, \quad k=1, \ldots, n .
$$

So the values of $g(x)$ are the breaking points of the flux $h(u)$. We can approximate the given initial data $v_{0}$ by taking a cell average, not just breaking points. Then the initial discretization $u_{0}$ can be written in the form of (6.8) with $\bar{t}_{k}=\infty$. This is a simplified version of the front tracking method under $(\mathrm{H})$.

Acknowledgments. The author would like to thank Professor A. E. Tzavaras. He gave the author the motivation and valuable remarks for this work. The author also would like to thank Professor Giovanni Russo and the anonymous referees for their helpful suggestions. He is also grateful to people in the Institute for Mathematics and its Applications (IMA) for helpful discussions and support.

\section{REFERENCES}

[1] A. L. Bertozzi, A. MünCh, ANd M. Shearer, Undercompressive shocks in thin film flows, Phys. D, 134 (1999), pp. 431-464.

[2] A. Bressan, Global solutions of systems of conservation laws by wave-front tracking, J. Math. Anal. Appl., 170 (1992), pp. 414-432.

[3] A. Bressan, Hyperbolic Systems of Conservation Laws. The One-Dimensional Cauchy Problem, Oxford Lecture Ser. Math. Appl. 20, Oxford University Press, Oxford, 2000.

[4] R. Courant, K. Friedrichs, and H. Lewy, Uber die partiellen Differenzengleichungen der mathematisches Physik, Math. Ann., 100 (1928), pp. 32-74.

[5] R. Courant, K. Friedrichs, And H. Lewy, On the partial difference equations of mathematical physics, IBM J. Res. Develop., 11 (1967), pp. 215-234.

[6] V. CvetKovic and G. Dagan, Transport of kinetically sorbing solute by steady random velocity in heterogenous porous formations, J. Fluid Mech., 265 (1994), pp. 189-215.

[7] C. M. Dafermos, Polygonal approximations of solutions of the initial value problem for a conservation law, J. Math. Anal. Appl., 38 (1972), pp. 33-41.

[8] C. M. Dafermos, Regularity and large time behaviour of solutions of a conservation law without convexity, Proc. Roy. Soc. Edinburgh Sect. A, 99 (1985), pp. 201-239. 
[9] C. M. Dafermos, Hyperbolic Conservation Laws in Continuum Physics, Grundlehren Math. Wiss. 325, Springer-Verlag, New York, 2000.

[10] J. GLimm, Solutions in the large for nonlinear hyperbolic systems of equations, Comm. Pure Appl. Math., 18 (1965), pp. 697-715.

[11] S. K. Godunov, A difference method for numerical calculation of discontinuous solutions of the equations of hydrodynamics, Mat. Sb. (N.S.), 47 (1959), pp. 271-306 (in Russian).

[12] H. Holden And L. Holden, On scalar conservation laws in one dimension, in Ideas and Methods in Mathematical Analysis, Stochastics, and Applications (Oslo, 1988), Cambridge Univ. Press, Cambridge, UK, 1992, pp. 480-509.

[13] H. Holden, K. A. Lie, and N. H. Risebro, An unconditionally stable method for the Euler equations, J. Comput. Phys., 150 (1999), pp. 76-96.

[14] H. Holden AND N. H. RisebRo, A method of fractional steps for scalar conservation laws without the CFL condition, Math. Comp., 60 (1993), pp. 221-232.

[15] L. HöRmander, Lectures on Nonlinear Hyperbolic Differential Equations, Math. Appl. 26, Springer-Verlag, Berlin, 1997.

[16] Y.-J. KIM, Asymptotic behavior of solutions to scalar conservation laws and optimal convergence orders to $N$-waves, J. Differential Equations, to appear.

[17] Y. J. Kim and A. E. Tzavaras, Diffusive $N$-waves and metastability in Burgers equation, SIAM J. Math. Anal., 33 (2001), pp. 607-633.

[18] S. N. KRuzhkov, First order quasilinear equations in several independent variables, Math. USSR Sb., 10 (1970), pp. 217-243.

[19] S. N. KruzhKov, First order quasilinear equations in several independent variables, Mat. Sb., 123 (1970), pp. 228-255.

[20] R. J. LeVeque, Large time step shock-capturing techniques for scalar conservation laws, SIAM J. Numer. Anal., 19 (1982), pp. 1091-1109.

[21] K.-A. LiE, Front tracking for one-dimensional quasilinear hyperbolic equations with variable coefficients, Numer. Algorithms, 24 (2000), pp. 275-298.

[22] K.-A. Lie, V. Haugse, and K. H. Karlsen, Dimensional splitting with front tracking and adaptive grid refinement, Numer. Methods Partial Differential Equations, 14 (1998), pp. 627-648.

[23] T.-P. LiU And M. PierRe, Source-solutions and asymptotic behavior in conservation laws, J. Differential Equations, 51 (1984), pp. 419-441.

[24] B. LuCIER, A moving mesh numerical method for hyperbolic conservation laws, Math. Comp., 46 (1986), pp. 59-69.

[25] K. Miller, Moving finite elements. II, SIAM J. Numer. Anal., 18 (1981), pp. 1033-1057.

[26] G. Whitham, Linear and Nonlinear Waves, Pure and Applied Mathematics, Wiley-Interscience, New York, 1974. 